\title{
Polyhedral techniques in combinatorial optimization II: applications and computations
}

Citation for published version (APA):

Aardal, K., \& van Hoesel, C. P. M. (1999). Polyhedral techniques in combinatorial optimization II: applications and computations. Statistica Neerlandica, 53(2), 131-177. https://doi.org/10.1111/14679574.00104

Document status and date:

Published: 01/01/1999

DOI:

10.1111/1467-9574.00104

Document Version:

Publisher's PDF, also known as Version of record

Document license:

Taverne

Please check the document version of this publication:

- A submitted manuscript is the version of the article upon submission and before peer-review. There can be important differences between the submitted version and the official published version of record.

People interested in the research are advised to contact the author for the final version of the publication, or visit the DOI to the publisher's website.

- The final author version and the galley proof are versions of the publication after peer review.

- The final published version features the final layout of the paper including the volume, issue and page numbers.

Link to publication

\footnotetext{
General rights rights.

- You may freely distribute the URL identifying the publication in the public portal. please follow below link for the End User Agreement:

www.umlib.nl/taverne-license

Take down policy

If you believe that this document breaches copyright please contact us at:

repository@maastrichtuniversity.nl

providing details and we will investigate your claim.
}

Copyright and moral rights for the publications made accessible in the public portal are retained by the authors and/or other copyright owners and it is a condition of accessing publications that users recognise and abide by the legal requirements associated with these

- Users may download and print one copy of any publication from the public portal for the purpose of private study or research.

- You may not further distribute the material or use it for any profit-making activity or commercial gain

If the publication is distributed under the terms of Article $25 \mathrm{fa}$ of the Dutch Copyright Act, indicated by the "Taverne" license above, 
Statistica Neerlandica (1999) Vol. 53, nr. 2, pp. 131-177

\title{
Polyhedral techniques in combinatorial optimization II: applications and computations
}

\author{
K. Aardal* \\ Department of Computer Science, Utrecht University, P.0. Box 80089, \\ 3508 TB Utrecht, The Netherlands \\ C. P. M. van Hoesel** \\ Department of Quantitative Economics, Limburg University, \\ P.O. Box 616, 6200 MD Maastricht, The Netherlands
}

\begin{abstract}
The polyhedral approach is one of the most powerful techniques available for solving hard combinatorial optimization problems. The main idea behind the technique is to consider the linear relaxation of the integer combinatorial optimization problem, and try to iteratively strengthen the linear formulation by adding violated strong valid inequalities, i.e., inequalities that are violated by the current fractional solution but satisfied by all feasible solutions, and that define highdimensional faces, preferably facets, of the convex hull of feasible solutions. If we have the complete description of the convex hull of feasible solutions at hand all extreme points of this formulation are integral, which means that we can solve the problem as a linear programming problem. Linear programming problems are known to be computationally easy. In Part 1 of this article we discuss theoretical aspects of polyhedral techniques. Here we will mainly concentrate on the computational aspects. In particular we discuss how polyhedral results are used in cutting plane algorithms. We also consider a few theoretical issues not treated in Part 1, such as techniques for proving that a certain inequality is facet defining, and that a certain linear formulation gives a complete description of the convex hull of feasible solutions. We conclude the article by briefly mentioning some alternative techniques for solving combinatorial optimization problems.
\end{abstract}

Key Words and Phrases: strong valid inequalities, facets, convex hull, cutting plane algorithm, branch-and-cut algorithm.

\section{Introduction}

A combinatorial optimization problem is an integer linear programming (ILP) problem

$$
\min \left\{c x: A x \leq b, x \in \mathbb{Z}^{n}\right\}
$$

\footnotetext{
*aardal@cs.uu.nl

** S.vanHoesel@KE.UniMaas.nl

(C) VVS, 1999. Published by Blackwell Publishers, 108 Cowley Road, Oxford OX4 1JF, UK and 350 Main Street, Malden, MA 02148, USA.
} 
having a combinatorial character. A well-known combinatorial optimization problem is the traveling salesman problem, i.e., the problem of finding the shortest tour through a number of cities such that each city is visited precisely once. The only known method for solving the traveling salesman problem is an enumerative method, such as branch-and-bound. If the lower bound obtained by solving the linear relaxation of the problem is not very close to the optimal value, then we can expect that the branchand-bound tree will grow too big to be manageable for any realistic instance. The idea behind polyhedral combinatorics is to obtain a strong lower bound on the optimal solution value by finding a good linear formulation of the set $X=\left\{x \in \mathbb{Z}^{n}: A x \leq b\right\}$ of feasible solutions. This is done by adding linear inequalities that are necessary in the description of the convex hull of $X$ to the original linear formulation $A x \leq b$. The convex hull of $X$ is the smallest convex set containing all points in $X$. The advantage with this approach is that if the convex hull of $X$ is known, we can solve $\min \{c x: x \in \operatorname{conv}(X)\}$ as a linear programming problem, which is computationally easy, but gives the same solution as optimizing over $X$.

As discussed in Part I of this article (AARdal and VAN Hoesel, 1996) it is hard in general to describe the convex hull of $X$ by concise families of inequalities even if we allow for classes containing exponentially many inequalities. In a practical setting, however, the complete description of the convex hull of $X$ is not needed. What is important is that we have a good description of the region close to the optimal solution, which suggests an approach where we generate linear inequalities as they are needed. Such an algorithm is usually called a cutting plane algorithm, and is typically embedded in a branch-and-bound procedure to produce good lower bounds. To make a cutting plane algorithm work we essentially need to consider three issues: (i) develop families of strong valid inequalities, (ii) develop separations algorithms, i.e., algorithms for identifying violated inequalities belonging to the various families, and (iii) designing an efficient implementation of the complete framework, including a branch-and-bound algorithm, a preprocessor, primal heuristics for finding good feasible solutions, and a branching strategy for the branch-and-bound phase.

In Part I we considered some important theoretical aspects of polyhedral combinatorics and cutting plane techniques. Questions that we were asking were for instance: Is there an algorithmic way to generate all inequalities necessary to describe the convex hull of feasible solutions? When can we expect to be able to describe the convex hull of feasible solutions using concise families of valid inequalities? How difficult is it to identify a violated linear inequality? In this article we shall mainly study computational aspects of polyhedral techniques, even though we study a few techniques and theoretical issues that were not treated in Part I.

When developing families of valid inequalities for a certain problem $P$ it is useful to consider valid inequalities for relaxations of $P$, since an inequality that is valid for a relaxation of $P$ is also valid for $P$. Once we gain insight in the facial structure of the simpler relaxation we can try to develop more problem specific inequalities. Moreover, we can include separation algorithms for valid inequalities for several common (C) VVS, 1999 
relaxations of integer and combinatorial optimization problems, and algorithms for automatically detecting the structure of these relaxations, as a basic feature in our cutting plane algorithm. In Part I we describe families of inequalities for a few generic combinatorial structures. In Section 2 we will make a more extensive presentation, and illustrate some useful techniques. One such technique is lifting, which is used if we have a valid inequality for a set of solutions projected onto a subspace. By using lifting on the inequality we obtain a valid inequality for the full space. We will also discuss two ways of proving that an inequality is facet defining, and how to prove that a given set of families of valid inequalities generates the convex hull of feasible solutions. We conclude the section by giving a partial survey of polyhedral results for combinatorial optimization problems.

Next to the theoretical work of developing good classes of valid inequalities and algorithms for identifying violated inequalities, there is a whole range of implementation issues that have to be considered in order to make cutting plane techniques work well. One such issue is preprocessing. Important elements of preprocessing are to reduce the size of the initial formulation by deleting unnecessary variables and constraints, and to reduce the size of the constraint coefficients to make the instance numerically more attractive. Due to logical implications it may also be possible to delete some variables, which reduces the size of the problem formulation.

When applying a cutting plane algorithm we in general end up in the situation where the current solution $x^{*}$ is not feasible and where we are unable to identify an inequality violated by $x^{*}$. We then have to start a branch-and-bound phase. In a branch-and-bound algorithm we must decide precisely how to create new subproblems, or nodes, in the search tree, as well as a suitable search strategy. It is also possible to add inequalities in every node of the tree, in which case we need to keep track of where in the tree the various inequalities are valid. Preprocessing and other implementation issues are discussed in Section 3. To illustrate the computational possibilities of polyhedral techniques we present computational results for some selected problem types in Section 4.

Even though polyhedral combinatorics has been the foremost tool for solving large instances of a vast collection of combinatorial optimization problems it is not the only technique available, and depending on the problem type it may be preferable to choose a different method. In Section 5, we briefly mention alternative approaches to solving integer and combinatorial optimization problems.

For the reader that is interested in studying polyhedral combinatorics in more detail, we recommend the following books and survey articles. The books by SchriJver (1986), and by NemHauser and Wolsey (1988) treat all aspects of polyhedral combinatorics as well as its links to linear and integer programming. The latter book also contains quite a number of problem specific results. The book by GRÖTSCHEL, Lovász and SCHRIJVER (1988) contains algorithmic results in polyhedral combinatorics derived from the theory of geometry of numbers. The survey article by JÜNGeR, ReINELt and ThIENEL (1995) treats several issues regarding implementations of cutting plane algorithms. Aardal and Weismantel (1997), and Caprara and (C) VVS, 1999 
FISCHETTI (1997) provide annotated bibliographies of polyhedral combinatorics and branch-and-cut algorithms respectively.

\section{Polyhedral results for generic combinatorial structures}

Some combinatorial structures occur as substructures in a large number of combinatorial optimization problems. The study of these generic structures has two purposes. First, a valid inequality for a generic structure may form the theoretical starting point for developing families of inequalities for more specialized problems. Second, valid inequalities for generic structures are often effective for more specialized problems as well, as implementing separation algorithms for generic inequalities is very useful in general-purpose cutting plane algorithms.

Here we present some well-known generic structures, extending our presentation in Part I. When discussing various families of inequalities we also take the opportunity to describe different techniques, such as facet proving techniques, techniques for proving that certain families of inequalities define the convex hull of feasible solutions, and lifting.

\subsection{Preliminaries}

Here we introduce basic definitions that are needed to understand the terminology used in subsequent sections.

The set of linear combinations of a set of vectors $x^{1} \ldots x^{K} \subset \mathbb{R}^{n}$ is the linear space $L S=\left\{\sum_{k=1}^{K} \alpha_{k} x^{k}: \alpha \in \mathbb{R}^{K}\right\}$. If $x^{1} \ldots x^{K}$ form a minimal system, i.e., none of the vectors is a linear combination of the others, then the vectors $x^{1} \ldots x^{K}$ are called linearly independent. Equivalently, the vectors $x^{1} \ldots x^{K}$ are linearly independent if $\alpha_{k}=0$, for all $k$, is the unique solution to the system $\sum_{k=1}^{K} \alpha_{k} x^{k}=0$. The dimension of a linear space $L S$, denoted by $\operatorname{dim}(L S)$ is defined as the maximum number of linearly independent points in the space.

The set of affine combinations of the $K+1$ points $x^{0}, x^{1} \ldots x^{K} \subset \mathbb{R}^{n}$ is called an affine space $A S=\left\{\sum_{k=0}^{K} \alpha_{k} x^{k}: \alpha \in \mathbb{R}^{K+1} ; \sum_{k=0}^{K} \alpha_{k}=1\right\}$. An affine space can be viewed as a linear space translated over a vector $x^{0}: A S=\left\{x^{0}+\sum_{k=1}^{K} \beta_{k}\left(x^{k}-x^{0}\right): \beta \in \mathbb{R}^{K}\right\}$. If the set of points $x^{0} \ldots x^{K}$ is a minimal system, i.e., none of the points is an affine combination of the others, then the points $x^{0} \ldots x^{K}$ are called affinely independent. Equivalently, the points $x^{0} \ldots x^{K}$ are affinely independent if $\alpha_{k}=0$, for all $k$, is the unique solution to the system $\Sigma_{k=0}^{K} \alpha_{k} x^{k}=0 ; \sum_{k=0}^{K} \alpha_{k}=0$. The dimension of an affine space, denoted by $\operatorname{dim}(A S)$, is the maximum number of affinely independent points minus 1 . Thus, if the points $x^{0} \ldots x^{K}$ are affinely independent, the affine space defined by these points has dimension $K$.

A polyhedron $P$ is the set of points satisfying a system of finitely many linear inequalities, i.e., $P=\left\{x \in \mathbb{R}^{n}: A x \leq b\right\}$. The dimension of $P$, denoted $\operatorname{dim}(P)$, is the dimension of a smallest (by inclusion) affine space containing $P$. A bounded polyhedron is called a polytope. 
An inequality $\pi x \leq \pi_{0}$ is called valid for a polyhedron $P$ if each point in $P$ satisfies the inequality. The set $F=\left\{x \in P: \pi x=\pi_{0}\right\}$ is called a face of $P$ and the valid inequality $\pi x \leq \pi_{0}$ is said to define the face $F$. A face is called proper if $\emptyset \neq F$ and $F \neq P$. The dimension of a proper face $F, \operatorname{dim}(F)$, is strictly smaller than $\operatorname{dim}(P)$. If $\operatorname{dim}(F)=\operatorname{dim}(P)-1$, i.e., if $F$ is maximal, we call $F$ a facet. The reason why we are interested in the facet defining inequalities is that they are precisely the inequalities that we need to describe the convex hull of feasible solutions, in addition to the set of inequalities that are satisfied with equality by every feasible point.

If $\pi x \leq \pi_{0}$ and $\gamma x \leq \gamma_{0}$ are two valid inequalities for a certain polyhedron $P \in \mathbb{R}_{+}^{n}$, then $\pi x \leq \pi_{0}$ dominates $\gamma x \leq \gamma_{0}$ if there exists $u>0$ such that $\pi \geq u \gamma$ and $\pi_{0} \leq u \gamma_{0}$, and $\left(\pi, \pi_{0}\right) \neq\left(u \gamma, u \gamma_{0}\right)$.

The convex hull of feasible solutions, denoted $\operatorname{conv}(X)$, is the set of points that can be obtained by taking convex combinations of points in $X$, i.e.,

$$
\operatorname{conv}(X)=\left\{\sum_{k=1}^{K} \lambda_{k} x^{k}: X=\left\{x^{k}\right\}_{k=1}^{K}, \quad \sum_{k=1}^{K} \lambda_{k}=1, \lambda_{k} \geq 0, k=1, \ldots, K\right\}
$$

Given a vector $x^{*}$, the separation problem based on a family $F$ of inequalities is the problem of finding an inequality $\pi x \leq \pi_{0}$ belonging to $F$ that is violated by $x^{*}$, i.e., $\pi x^{*}>\pi_{0}$, or providing a proof that no such inequality in $F$ exists. An algorithm for solving the separation problem is called a separation algorithm.

\subsection{The vertex packing problem}

Here we describe two classes of valid inequalities for the vertex packing problem. We also give an example of an easy facet proof and illustrate lifting techniques. Lifting is an iterative technique where we start with an inequality that is valid under the condition that a subset $N$ of the variables are fixed. At each iteration a subset $M \subseteq N$ of the fixed variables are included in the inequality with coefficients that guarantee that the resulting inequality is valid. In sequential lifting the set $M$ consists of one variable at each iteration, whereas in simultaneous lifting there are no restrictions on the choice of $M$. Typically we have $M=N$.

Consider an undirected graph $G=(V, E)$ where $V$ is the set of vertices and $E$ the set of edges, i.e., unordered pairs of vertices. A vertex packing in $G$ is a subset $V^{\prime} \subseteq V$ of vertices such that no two vertices in $V^{\prime}$ are adjacent. We define variables $x_{v}$ for each vertex $v$, and let $x_{v}=1$ if $v \in V^{\prime}$ and $x_{v}=0$ otherwise. The integer programming formulation of the maximum cardinality vertex packing problem is given below

$$
\begin{array}{lll}
\max & \sum_{v \in V} x_{v} & \\
\text { s.t. } & x_{v}+x_{w} \leq 1 & \text { for all }\{v, w\} \in E \\
& x_{v} \in\{0,1\} & \text { for all } v \in V
\end{array}
$$


The vertex packing problem is sometimes referred to as the independent set problem or the stable set problem. Let $X_{V P^{G}}$ be the set of incidence vectors corresponding to feasible vertex packings in the graph $G$, and let $\alpha(G)$ be the maximum cardinality of a vertex packing in $G$. An edge is called critical if its removal from $G$ produces a graph $G^{\prime}$ with $\alpha\left(G^{\prime}\right)>\alpha(G)$. ChvátAL (1975) derived the following general sufficient condition for an inequality to define a facet of $\operatorname{conv}\left(X_{V P^{G}}\right)$.

Theorem 1 Chvítal (1975). Let $E^{*}$ be the set of critical edges of $G$. If the graph $G^{*}=\left(V, E^{*}\right)$ is connected, then the inequality $\sum_{j \in V} x_{j} \leq \alpha(G)$ defines a facet of $\operatorname{conv}\left(X_{V P^{G}}\right)$.

A clique in a graph $G$ is a subgraph of $G$ where each two vertices are connected by an edge, see Figure 1a. A clique is maximal if it is not contained in any other clique. Since no two vertices in $V^{\prime}$ are allowed to be adjacent we could take any clique $C$ in $G$ and require that at most one vertex belonging to $C$ should belong to the vertex packing $V^{\prime}$ giving the valid inequality

$$
\sum_{j \in C} x_{j} \leq 1
$$

Theorem 2 PAdBerg (1973). Let C be a clique in the graph G. The clique inequality (4) defines a facet of $\operatorname{conv}\left(X_{V P^{G}}\right)$ if and only if $C$ is maximal.

Proof. Sufficiency: The dimension of the vertex packing polytope is $|V|$. Hence, to prove that (4) defines a facet of $\operatorname{conv}\left(X_{V P^{G}}\right)$ we need to find $|V|$ affinely independent points that are tight for (4). Let $C$ be a maximal clique. For every $v \in C$ we take the vertex packing that contains only $v$. For $v \notin C$ we first choose a node $w \in C$ that is not adjacent to $v$. Since $C$ is maximal such a node exists. We then take the vertex packing that contains both nodes $v$ and $w$. The $|V|$ points given above are feasible, satisfy the clique inequality with equality, and are affinely independent. Thus, the inequality is facet-defining.

Necessity: If $C$ is not maximal then there is a clique $C^{\prime}$ such that $C \subset C^{\prime}$. The clique inequality defined by $C^{\prime}$ dominates the inequality defined by $C$.

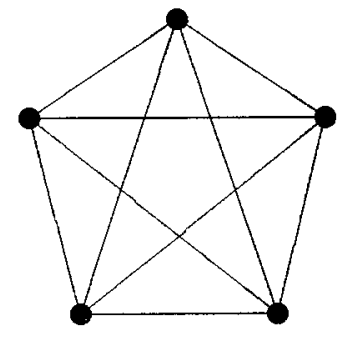

a)

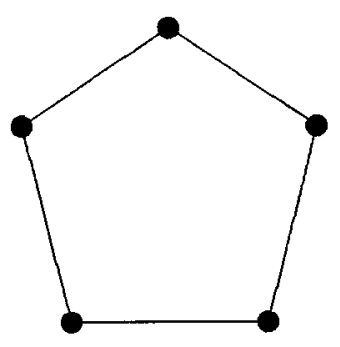

b)

Fig. 1. a) A clique. b) An odd hole.

(C) VVS, 1999 
Another class of valid inequalities for the vertex packing problem is the family of odd-hole inequalities. An odd hole $H$ in a graph $G$ is a chordless cycle consisting of an odd number of vertices, i.e., there are no edges of $G$ connecting any nonconsecutive vertices in $H$, see Figure $1 \mathrm{~b}$. Since the number of vertices in $H$ is odd, at most $[|H| /$ 2] $=(|\mathrm{H}|-1) / 2$ vertices in $H$ can belong to any vertex packing. Hence the following odd-hole inequality is valid.

$$
\sum_{j \in H} x_{j} \leq \frac{|H|-1}{2}
$$

PAdBerg (1973) showed that (5) defines a facet of $\operatorname{conv}\left(X_{V P^{G}} \cap\left\{x \in\{0,1\}^{|V|}: x_{j}=0\right.\right.$ for all $j \notin H\}$ ), i.e., in general (5) defines a face of $\operatorname{conv}\left(X_{V P^{G}}\right)$ of dimension less than $\operatorname{dim}\left(X_{V P^{G}}\right)-1$. The question is whether it is possible to increase the dimension of (5) such that (5) becomes a facet for $\operatorname{conv}\left(X_{V P^{G}}\right)$. One way of increasing this dimension is through sequential lifting (PADBERG, 1973, and WolseY, 1976a), which is illustrated in the following example.

EXAMPLE 1 The graph in Figure 2 is an odd hole with a central vertex adjacent to all vertices of the hole. This structure, called a wheel, is used to illustrate the sequential lifting procedure. The inequality is $x_{1}+x_{2}+x_{3}+x_{4}+x_{5} \leq 2$ defines a facet of $\operatorname{conv}\left(X_{V P^{G}} \cap\left\{x \in\{0,1\}^{6}: x_{6}=0\right\}\right)$. The problem is to determine the maximum nonnegative value of the constant $\alpha$ such that $x_{1}+x_{2}+x_{3}+x_{4}+x_{5}+\alpha x_{6} \leq 2$ is a valid inequality of $\operatorname{conv}\left(X_{V P^{G}}\right)$. If $x_{6}=0, \alpha$ can take any value, hence assume that $x_{6}=1$. If $x_{6}=1$ we must have $x_{j}=0, j=1, \ldots, 5$, since $x_{6}$ is adjacent to all other vertices. Hence, the maximal value of $\alpha$ is $\alpha=2$. The following two theorems imply that if the inequality is facet defining in the reduced space, and if we "lift" in all variables sequentially with maximal coefficients, then the resulting inequality defines a facet in the full space.

Theorem 3 Wolsey (1976a). Let $S \subseteq\{0,1\}^{n}$. Suppose

$$
\sum_{j=2}^{n} \pi_{j} x_{j} \leq \pi_{0}
$$

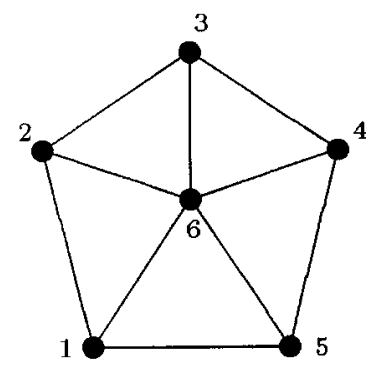

Fig. 2. A wheel.

(C) VVS, 1999 
is valid for $S^{0}=S \cap\left\{x \in\{0,1\}^{n}: x_{1}=0\right\}$. If $S \cap\left\{x \in\{0,1\}^{n}: x_{1}=1\right\} \neq \emptyset$, then

$$
\alpha x_{1}+\sum_{j=2}^{n} \pi_{j} x_{j} \leq \pi_{0}
$$

is valid for $S$ for any $\alpha \leq \pi_{0}-\max _{S \cap\left\{x: x_{1}=1\right\}}\left\{\sum_{j=2}^{n} \pi_{j} x_{j}\right\}$. If $(6)$ defines a face of $\operatorname{conv}\left(S^{0}\right)$ of dimension $k$, and if $\alpha$ is chosen maximal, then (7) defines a face of $\operatorname{conv}(S)$ of dimension $k+1$.

Theorem 4 Wolsey (1976a). Let $S \subseteq\{0,1\}^{n}$. Suppose

$$
\sum_{j=2}^{n} \pi_{j} x_{j} \leq \pi_{0}
$$

is valid for $S^{1}=S \cap\left\{x \in\{0,1\}^{n}: x_{1}=1\right\}$. If $S \cap\left\{x \in\{0,1\}^{n}: x_{1}=0\right\} \neq \emptyset$, then

$$
\beta x_{1}+\sum_{j=2}^{n} \pi_{j} x_{j} \leq \pi_{0}+\beta
$$

is valid for $S$ for any $\beta \geq \max _{S \cap\left\{x: x_{1}=0\right\}} \sum_{j=2}^{n} \pi_{j} x_{j}-\pi_{0}$. If $(8)$ defines a face of conv $\left(S^{1}\right)$ of dimension $k$, and if $\beta$ is chosen minimal, then (9) defines a face of conv $(S)$ of dimension $k+1$.

Sequential lifting is sequence dependent, such that different lifting sequences give rise to different inequalities. ZEMEL (1978) proposed a more general lifting procedure, called simultaneous lifting. As the name indicates, the coefficients of several variables are determined simultaneously, yielding inequalities that in general cannot be obtained by sequential lifting. For notational ease we consider the case where a set of variables that are all fixed to zero are simultaneously lifted. Let $S \subseteq\{0,1\}^{n}$, and suppose that the inequality

$$
\sum_{j=k+1}^{n} \pi_{j} x_{j} \leq \pi_{0}
$$

is valid for $S^{0}=S \cap\left\{x \in\{0,1\}^{n}: x_{1}=x_{2}=\cdots=x_{k}=0\right\}$. If

$$
S \cap\left\{x \in\{0,1\}^{n}: \sum_{j=1}^{k} x_{j} \geq 1\right\} \neq \emptyset
$$

then

$$
\sum_{j=1}^{k} \alpha_{j} x_{j}+\sum_{j=k+1}^{n} \pi_{j} x_{j} \leq \pi_{0}
$$

is valid for $S$ if for any $\left(x_{1}^{\prime}, \ldots, x_{k}^{\prime}\right) \neq 0$, we have that $\sum_{j=1}^{k} \alpha_{j} x_{j}^{\prime} \leq \pi_{0}-$ $\max _{S \cap\left\{x: x_{1}=x_{1}^{\prime}, \ldots, x_{k}=x_{k}^{\prime}\right\}}\left\{\sum_{j=k+1}^{n} \pi_{j} x_{j}\right\}$. Hence, the feasible vectors $\alpha=\left(\alpha_{1}, \ldots, \alpha_{k}\right)$ define (c) VVS, 1999 

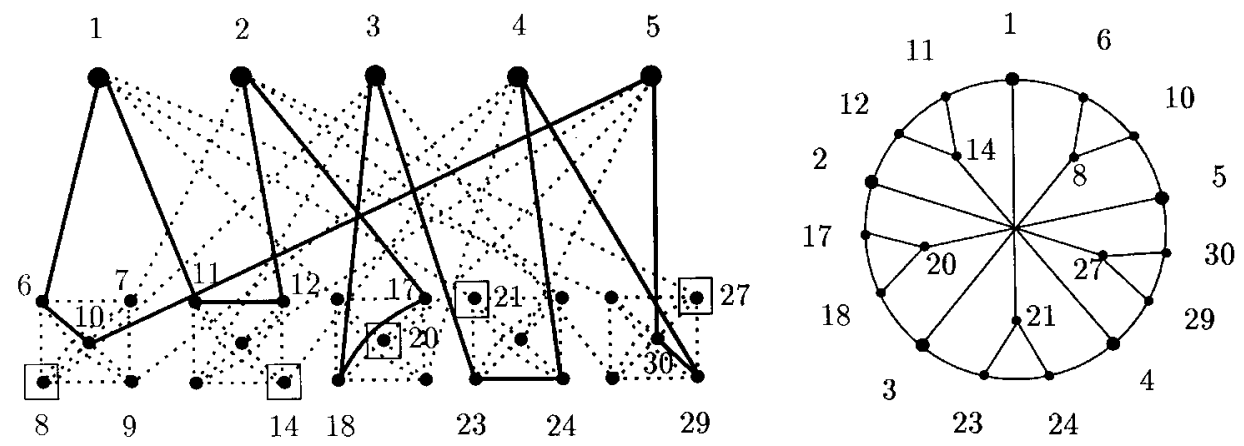

Fig. 3. Simultaneous lifting of an odd-hole inequality.

a polyhedral set. The extreme points of this set yield new facet defining inequalities in general if the original inequality is facet defining. For more details, see ZEMEL (1978). We will give an example of a vertex packing problem where simultaneous lifting of an odd-hole inequality yields an inequality that cannot be obtained by sequential lifting.

EXAMPLE 2 The left part of Figure 3 shows the original underlying graph in dotted lines, and the odd hole in solid lines. The odd-hole inequality is:

$$
\begin{aligned}
x_{1} & +x_{6}+x_{10}+x_{5}+x_{30}+x_{29}+x_{4}+x_{24}+x_{23}+x_{3}+x_{18} \\
& +x_{17}+x_{2}+x_{12}+x_{11} \leq 7
\end{aligned}
$$

The right figure illustrates the structure corresponding to the inequality that is obtained by simultaneously lifting the variables corresponding to vertices $8,14,20,21$ and 27 . These are the vertices surrounded by squares in the left figure. The coefficients of all lifted variables are equal to one half. The resulting inequality is facet defining and cannot be obtained by sequential lifting.

$$
\begin{aligned}
& x_{1}+x_{6}+x_{10}+x_{5}+x_{30}+x_{29}+x_{4}+x_{24}+x_{23}+x_{3}+x_{18}+x_{17} \\
& +x_{2}+x_{12}+x_{11}+\frac{1}{2}\left(x_{8}+x_{14}+x_{20}+x_{21}+x_{27}\right) \leq 7
\end{aligned}
$$

If we apply sequential lifting to the same initial odd-hole inequality we obtain the lifting illustrated in Figure 4 or any of the four liftings that can be obtained by rotating the figure. The corresponding facet-defining inequality is:

$$
\begin{aligned}
& x_{1}+x_{6}+x_{10}+x_{5}+x_{30}+x_{29}+x_{4}+x_{24}+x_{23}+x_{3}+x_{18} \\
& +x_{17}+x_{2}+x_{12}+x_{11}+\left(x_{8}+x_{21}\right) \leq 7
\end{aligned}
$$

For more details on lifting procedures, see NemHAuser and Wolsey (1988). (C) VVS, 1999 


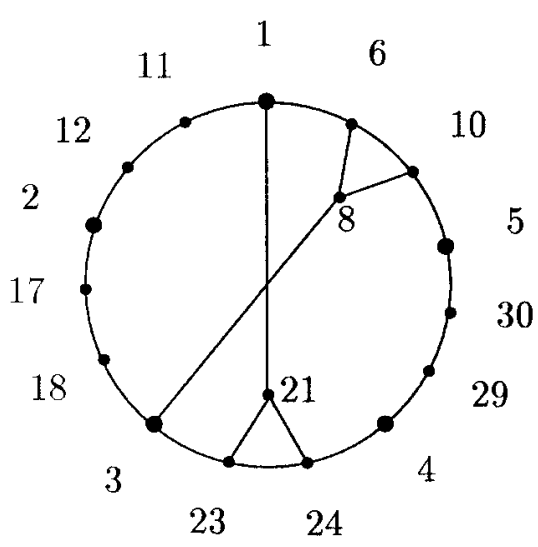

Fig. 4. Sequential lifting of an odd-hole inequality.

\subsection{The knapsack problem}

Let $N=\{1, \ldots, n\}$. The knapsack problem is formulated as

$$
\begin{array}{ll}
\max & \sum_{j \in N} c_{j} x_{j} \\
\text { s.t. } & \sum_{j \in N} a_{j} x_{j} \leq b \\
& x_{j} \in\{0,1\} \quad \text { for all } j \in N
\end{array}
$$

The knapsack problem occurs as a substructure of several combinatorial optimization problems having a capacity or budget constraint. Assume that the vectors $c, a$ and the right-hand side $b$ are integral, and let $X_{K}$ be the set of incidence vectors corresponding to the feasible solutions to the knapsack problem. Let $C$ be a subset of $N$ such that $\sum_{j \in C} a_{j}>b$, and such that $C$ is minimal with respect to this property, i.e., $\sum_{j \notin S} a_{j} \leq b$ for all $S \subset C$. We call the set $C$ a minimal cover with respect to $N$ and $b$. In Part I we described the family of knapsack cover inequalities (BALAs, 1975, HAMMER et al., 1975, and WolseY, 1975)

$$
\sum_{j \in C} x_{j} \leq|C|-1
$$

The inequalities (17) are valid for $X_{K}$ since, if we include all items in $C$ in the knapsack, we exceed the right-hand side $b$, which means that we have to exclude at least one of the elements in $C$.

In Part I we discussed the special case of a lifted cover inequality that is obtained if we consider the extension $E(C)$ of a minimal cover $C$, where $E(C)=C \cup\left\{k \in N \backslash C: a_{k} \geq a_{j}\right.$, for all $\left.j \in C\right\}$. The lifting coefficients of the variables in $E(C) \backslash C$ are all equal to one. The most general form of the knapsack cover inequality is obtained by partitioning the set $N$ into the sets $\left(N^{\prime}, N \backslash N^{\prime}\right)$. Let $x_{j}=0$ for all $j \in N \backslash N^{\prime}$, and let $C^{\prime}$ be a minimal cover with respect to $N^{\prime}$ and $b-\sum_{j \in N^{\prime} \backslash C^{\prime}} a_{j}$. (c) VVS, 1999 
Moreover, let $x_{j}=1$ for all $j \in N^{\prime} \backslash C^{\prime}$. By using the lifting results presented in Theorems 3 and 4, we can conclude that $\operatorname{conv}\left(X_{K}\right)$ has a facet of the following form

$$
\sum_{j \in N \backslash N^{\prime}} \alpha_{j} x_{j}+\sum_{j \in N^{\prime} \backslash C^{\prime}} \beta_{j} x_{j}+\sum_{j \in C^{\prime}} x_{j} \leq\left|C^{\prime}\right|-1+\sum_{j \in N^{\prime} \backslash C^{\prime}} \beta_{j}
$$

where $\alpha_{j} \geq 0$ for all $j \in N \backslash N^{\prime}$ and $\beta_{j} \geq 0$ for all $j \in N^{\prime} \backslash C^{\prime}$. Balas (1975) characterized the lifting coefficients $\alpha_{j}$ in the case where $N^{\prime} \backslash C^{\prime}=\emptyset$.

The family of $(1, k)$-configuration inequalities (PADBERG, 1980) is defined as follows. Let $\bar{C} \subseteq N$, and $t \in N \backslash \bar{C}$ be such that $\Sigma_{j \in \bar{C}} a_{j} \leq b$ and such that $Q \cup\{t\}$ is a minimal cover for all $Q \subseteq \bar{C}$ with $|Q|=k$. Let $T(r) \subseteq C$ vary over all subsets of cardinality $r$ of $\bar{C}$, where $r$ is an integer satisfying $k \leq r \leq|\bar{C}|$. The $(1, k)$-configuration inequality

$$
(r-k+1) x_{t}+\sum_{j \in T(r)} x_{j} \leq r
$$

is valid for $\operatorname{conv}\left(X_{K}\right)$. If $k=|\bar{C}|$ the knapsack cover inequality (17) is obtained. The $(1, k)$-configuration inequality (19) can be obtained by the following lifting procedure. We start with the inequality

$$
\sum_{j \in T(r)} x_{j} \leq k-1
$$

which is valid for $X_{K} \cap\left\{x \in\{0,1\}^{n}: x_{t}=1\right\}$. The maximal lifting coefficient of variable $x_{t}$ is equal to $r-k+1$.

In the following example we demonstrate how a $(1, k)$-configuration inequality is obtained.

ExAmple 3 Let $N=\{1, \ldots, 5\}$ and consider the set of vectors $\left\{x \in\{0,1\}^{5}: 15 x_{1}+\right.$ $\left.17 x_{2}+18 x_{3}+21 x_{4}+22 x_{5} \leq 52\right\}$. Let $\bar{C}=\{1,2,3\}$, and let $t=4$. We see that $\Sigma_{j \in \bar{C}} a_{j} \leq 52$ and that $Q \cup\{4\}$ defines a cover with respect to $N$ and $b$ for all $Q \subseteq \bar{C}$ with $|Q|=2$. First, let $r=2$. We then obtain the valid inequalities $x_{4}+x_{1}+x_{2} \leq 2$, $x_{4}+x_{1}+x_{3} \leq 2$ and $x_{4}+x_{2}+x_{3} \leq 2$. By letting $r=3$ we obtain $2 x_{4}+x_{1}+x_{2}+x_{3} \leq 3$.

\subsection{The fixed charge uncapacitated flow problem}

Here we consider a general class of valid inequalities for the uncapacitated fixedcharge flow polytope. Consider a directed graph $G=(V, A)$, and let $x_{i j}$ denote the flow on $\operatorname{arc}(i, j) \in A$. If $\operatorname{arc}(i, j)$ is used we have to pay a fixed cost. Therefore, let $y_{i j}=1$ if $\operatorname{arc}(i, j)$ is opened, and let $y_{i j}=0$ otherwise. Each node $i$ has a known outflow $d_{i}$. If $d_{i}$ is negative it means that node $i$ has an inflow. We use $d_{i}^{+}$to denote (c) VVS, 1999 
$\max \left\{0, d_{i}\right\}$. The polytope $X_{U F C}$ is defined as the set of vectors corresponding to the solutions satisfying the following constraints.

$$
\begin{array}{ll}
\sum_{\{k:(k, i) \in A\}} x_{k i}-\sum_{\{j:(i, j) \in A\}} x_{i j}=d_{i} & \text { for all } i \in V \\
0 \leq x_{i j} \leq M y_{i j} & \text { for all }(i, j) \in A \\
y_{i j} \in\{0,1\} & \text { for all }(i, j) \in A
\end{array}
$$

Constraints (21) are flow conservation constraints, and in constraint (22) $M$ is a large enough positive number, which we need in order to enforce $y_{i j}=1$ if $x_{i j}>0$. Typically $M=\sum_{i \in V} d_{i}^{+}$.

Let $X \subset V$, and let $E(X)$ be the subset of arcs for which both endpoints belong to $X$, i.e., $E(X)=\{(i, j) \in A: i, j \in X\}$. Moreover, let $(X, \bar{X})=(X, V \backslash X)=\{(i$, $j) \in A: i \in X, j \in V \backslash X\}$. Consider the subset $C \subseteq(\bar{X}, X)$, and the subset $R \subseteq E(X)$, and let $H=\{j:(i, j) \in C\}$. Let $V_{j}^{R}=\{j\} \cup\{k \in X$ : there exists a directed path from $j$ to $k$ using only arcs of $R\}$ for $j \in H$. The flow model is illustrated in Figure 5.

The following family of valid inequalities for $X_{U F C}$ was developed by VAN RoY and WOLSEY (1985).

$$
\sum_{(i, j) \in C} x_{i j} \leq \sum_{(i, j) \in C}\left(\sum_{k \in V_{j}^{R}} d_{k}^{+}\right) y_{i j}+\sum_{(i, j) \in E(X) \backslash R} x_{i j}+\sum_{(i, j) \in(X, \bar{X})} x_{i j}
$$

The intuition behind the inequalities is as follows. The flow on the arcs in the subset $C \subseteq(\bar{X}, X)$ either flows along $\operatorname{arcs}$ in $R$, or $\operatorname{arcs}$ in $E(X) \backslash R$ or $\arcsin (X, \bar{X})$. For $j \in X$ and $(i, j) \in C$, the part of the flow $x_{i j}$ that goes along arcs in $R$ is limited by the outflow $\Sigma_{k} d_{k}^{+}$, since the set $V_{j}^{+}$is defined as the nodes of $X$ that can be reached from $j$ by using arcs in $R$ only. This explains the coefficient of the $y_{i j}$-variables. A subclass of the inequalities $(24)$ is the family of $(l, S)$-inequalities for the economic lot-sizing problem presented in Section 2.6.1.

The separation problem based on the network inequalities (24) is difficult in general as we need to simultaneously choose sets $X, R$ and $C$. Van Roy and Wolsey treated

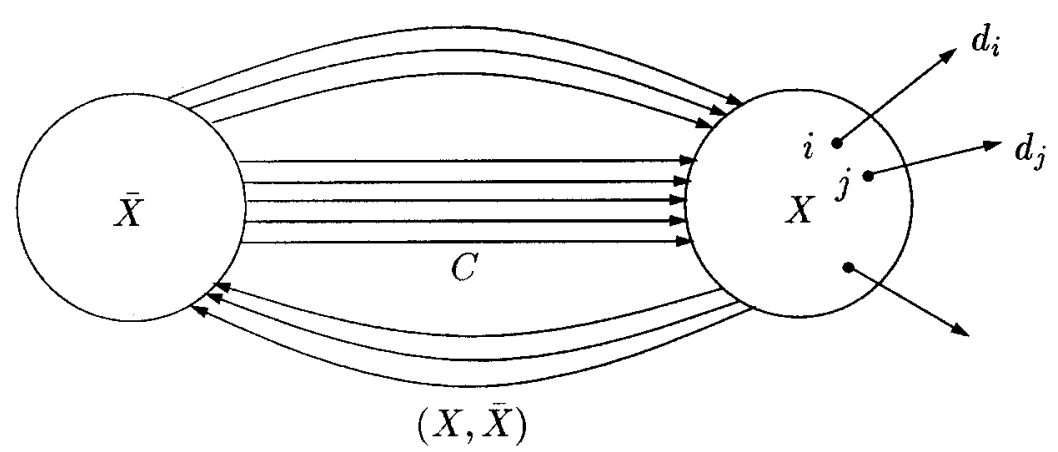

Fig. 5. The fixed charge uncapacitated flow model. 
three special cases where it is possible to generate a violated network inequality (24), or a weakened version of it, in polynomial time. Let $\left(x^{*}, y^{*}\right)$ denote a fractional solution. The easiest case is when the sets $X$ and $R$ are known and we only need to identify a set $C$. Here we only have to evaluate $\Sigma_{(i, j) \in(\bar{X}, X)}\left(x_{i j}^{*}-\left(\Sigma_{k \in V_{i}^{R}} d_{k}^{+}\right) y_{i j}^{*}\right)$, and let $C=\left\{(i, j) \in(\bar{X}, X): x_{i j}^{*}>\left(\Sigma_{k \in V^{R}} d_{k}^{+}\right) y_{i j}^{*}\right\}$. In the second case the set $C^{\prime}$ is fixed and we look for a set $X$ assuming that $R=E(X)$. The best choice of $X$ can be found using a maximum flow algorithm The third case deals with a slight modification of (24) developed for the generalization of the flow model (21)-(23) in which we have arcs with an upper bound, i.e., $0 \leq x_{k l} \leq m_{k l}$ for $(k, l) \in(E(X) \backslash R) \cup(X, \bar{X})$. In the modified inequality we want to replace $d_{k}^{+}$with $\left(d_{k}+m_{k l}\right)^{+}$for $(k$, $l) \in(E(X) \backslash R) \cup(X, \bar{X})$ and then remove the arc $x_{k l}$ from the inequality. Assume we have replaced the arcs in the subset $Q \subseteq(E(X) \backslash R) \cup(X, \bar{X})$. We then obtain the following valid inequality:

$$
\sum_{(i, j) \in C} x_{i j} \leq \sum_{(i, j) \in C} y_{i j}\left(\sum_{k \in V_{j}^{R}}\left(d_{k}^{+} \sum_{\{l:(k, l) \in Q\}} m_{k l}\right)\right)+\sum_{(i, j) \in E(X) \backslash(R \cup Q)} x_{i j}+\sum_{(i, j) \in(X, \bar{X}) \backslash Q} x_{i j}
$$

If the sets $X$ and $R$ are fixed, it is possible to find the best choice of $Q$ and $C$ in polynomial time.

\subsection{The single-node capacitated flow problem}

Consider a single node in a directed graph, and let $N$ be the set of arcs entering the node. The outflow from the node is equal to $b$. Let $x_{j}$ be a continuous variable denoting the flow on $\operatorname{arc} j$, and let $m_{j}$ be the capacity on $\operatorname{arc} j$. If $\operatorname{arc} j$ is open, then $y_{j}=1$, otherwise $y_{j}=0$. The following fixed charge single-node flow structure is a relaxation of many combinatorial flow models.

$$
\begin{array}{ll}
\sum_{j \in N} x_{j}=b & \\
0 \leq x_{j} \leq m_{j} y_{j} & \text { for all } j \in N \\
y_{j} \in\{0,1\} & \text { for all } j \in N
\end{array}
$$

Let $X_{F C}$ denote the set of vectors corresponding to feasible solutions to (26)-(28). In this section we will discuss the following topics. First, we will describe the basic flow cover inequality that is valid for $X_{F C}$, and show that this inequality is facet defining. We will use a different proof technique compared to the one used to prove Theorem 2. Next, we will discuss the separation problem based on the family of flow cover inequalities. Once we have a flow cover inequality we can extend it. We describe the result that if $m_{j}=m$ for all $j \in N$, then the extended flow cover inequalities, together with the defining inequalities define the convex hull of feasible solutions. Moreover, if all capacities are equal, then the extended flow cover inequalities can be separated in polynomial time. An application of the flow cover inequalities is shown in Section 2.6.2. 


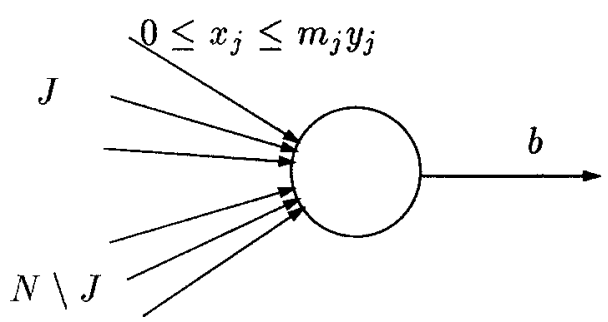

Fig. 6. The single-node capacitated flow model with sets $J$ and $N \backslash J$.

\subsubsection{The flow cover inequalities}

A subset $J \subseteq N$ is called a flow cover with respect to $N$ and $b$ if $\Sigma_{j \in J} m_{j}=b+\lambda$ with $\lambda>0$. If we have a flow cover $J$, and if all arcs $j \in J$ are open, i.e., $y_{j}=1$ for all $j \in J$, then $\Sigma_{j \in J} x_{j} \leq b$ since the total outflow is equal to $b$. If, however, we close one arc $k \in J, \quad$ then $\quad \sum_{j \in J \backslash\{k\}} x_{j} \leq \min \left\{b, \quad \sum_{j \in J} m_{j}-m_{k}\right\}=\min \left\{b, \quad b-\left(m_{k}-\lambda\right)\right\}=$ $b-\left(m_{k}-\lambda\right)^{+}$, yielding the valid inequality

$$
\sum_{j \in J} x_{j} \leq b-\sum_{j \in J}\left(m_{j}-\lambda\right)^{+}\left(1-y_{j}\right)
$$

In Figure 6 we illustrate the single-node capacitated flow model.

Theorem 5 Padberg, Van Roy and Wolsey (1985). Assume that $\Sigma_{j \in N} m_{j}-m_{r}>b$ for all $r \in N$, and that $J \subset N$. The flow cover inequality (29) defines a facet of conv $\left(X_{F C}\right)$ if and only if $\max _{j \in J}\left\{m_{j}\right\}>\lambda$.

Proof. Sufficiency: To prove that inequality (29) defines facets under the given conditions we will use a different technique than the one we use to prove Theorem 2 . The method used here is often referred to as the indirect method, see NEMHAUSER and Wolsey (1988), Chapter I.4, Theorem 3.6.

To show that inequality (29) is facet defining we need to show that (29) does not define an improper face, i.e., that there exists a feasible point such that inequality (29) is satisfied with strict inequality at this point. We also need to prove that the only inequality that is satisfied with equality by all points $(x, y) \in X_{F C}$ that lie on the hyperplane $\Sigma_{j \in J} x_{j}=b-\Sigma_{j \in J}\left(m_{j}-\lambda\right)^{+}\left(1-y_{j}\right)$, is inequality (29) plus $\gamma\left(\sum_{j \in N} x_{j}=b\right)$, where $\gamma$ is an arbitrarily chosen scalar. If there were more inequalities of this sort it would mean that these inequalities all define faces of lower dimension. Also, note that the polytope $\operatorname{conv}\left(X_{F C}\right)$ is not full-dimensional as all feasible points satisfy $\Sigma_{j \in N} x_{j}=b$. Therefore, each facet is uniquely represented up to a scalar multiple of this equality constraint. This is the reason why we add $\gamma\left(\sum_{j \in N} x_{j}=b\right)$ to inequality (29) in the facet proof. 
To see that inequality (29) does not define an improper face, we consider any feasible point for which the following holds.

$$
\begin{aligned}
y_{j} & =1 \quad \text { for all } j \in N \\
\sum_{j \in J} x_{j} & =b-\varepsilon \\
\sum_{j \in N \backslash J} x_{j} & =\varepsilon
\end{aligned}
$$

where $\varepsilon>0$ is a sufficiently small real number. Such a point is possible to construct since $J$ defines a cover, and since $J$ is a proper subset of $N$. Since inequality (29) is satisfied with strict inequality at this point, we have shown that inequality (29) does not define an improper face.

Next, we need to show that if all points $(x, y) \in X_{F C}$ that are tight for (29) satisfy

$$
\sum_{j \in N} \alpha_{j} x_{j}+\sum_{j \in N} \beta_{j} y_{j}=\alpha_{0}
$$

then
(i) $\beta_{j}=0$
for all $j \in N \backslash J$
(ii) $\alpha_{j}=\gamma$ for all $j \in N \backslash J$
(iii) $\alpha_{j}=\bar{\alpha}+\gamma$ for all $j \in J$
(iv) $\beta_{j}=-\bar{\alpha}\left(m_{j}-\lambda\right)^{+}$ for all $j \in J$
(v) $\alpha_{0}=\bar{\alpha}\left(b-\Sigma_{j \in J}\left(m_{j}-\lambda\right)^{+}\right)+\gamma b$.

To show that $\beta_{j}=0$ for all $j \in N \backslash J$, we consider any feasible solution in which $\Sigma_{j \in J} x_{\mathrm{j}}=b$, and $y_{j}=1$ for all $j \in J$ and all $(N \backslash J) \backslash\{l\}$, where $l$ is chosen arbitrarily. First, let $y_{l}=0$, and then create a new solution where every variable takes the same value as in the first solution, except $y_{l}$, which now takes value one. Evaluating (30) at both solutions and comparing the two expressions gives $\beta_{l}=0$. Since arc $l$ was chosen arbitrarily in $N \backslash J$, we can conclude that $\beta_{j}=0$ for all $j \in N \backslash J$.

Next, we show that $\alpha_{j}=\gamma$ for all $j \in N \backslash J$. Here we first consider a solution in which we close the arc $k \in J$ with largest capacity. Due to the assumptions of the theorem we know that $m_{k}>\lambda$. Let $y_{j}=1$ for all $j \in N \backslash\{k\}, \Sigma_{j \in J \backslash k} x_{j}=b-\left(m_{k}-\lambda\right)$, and let $\Sigma_{j \in N \backslash J} x_{j}=\left(m_{k}-\lambda\right)$. Furthermore, assume that $\varepsilon<x_{j}<m_{j}$ for all $j \in N \backslash J$, and for $\varepsilon>0$ sufficiently small. The second solution we consider is constructed as follows. Choose arbitrarily two arcs $j^{\prime}, j^{\prime \prime} \in N \backslash J$. Let all variables take the same values as in the first point except that we increase the flow by $\varepsilon$ on arc $j^{\prime}$ and decrease the flow by $\varepsilon$ on arc $j^{\prime \prime}$. Comparing the expressions obtained by evaluating (30) at the two solutions gives $\alpha_{j^{\prime}}=\alpha_{j^{\prime \prime}}$. Since $j^{\prime}$ and $j^{\prime \prime}$ were arbitrarily chosen, we have $\alpha_{j}=\gamma$ for all $j \in N \backslash J$.

To show that $\alpha_{j}=\bar{\alpha}+\gamma$ for all $j \in J$ we consider a solution in which all $\operatorname{arcs}$ in $N$ are open, and in which $\Sigma_{j \in J} x_{j}=b$ and $0<x_{j}<m_{j}$ for all $j \in J$. Now we can choose (c) VVS, 1999 
any two $\operatorname{arcs} j^{\prime}, j^{\prime \prime} \in J$ and make an $\varepsilon$-change of flow as in the previous step of the proof. This shows that $\alpha_{j^{\prime}}=\alpha_{j^{\prime \prime}}$. Varying over all choices of $j^{\prime}, j^{\prime \prime}$ gives $\alpha_{j}=\alpha^{1}$ for all $j \in J$. Assume that $\alpha^{1}=\bar{\alpha}+\gamma$. If $\bar{\alpha}=0$, the conditions (i)-(v) would be satisifed trivially. Hence, assume that $\bar{\alpha} \neq 0$.

We have now reduced equality (30) to the following expression

$$
(\bar{\alpha}+\gamma) \sum_{j \in J} x_{j}+\gamma \sum_{j \in N \backslash J} x_{j}+\sum_{j \in J} \beta_{j} y_{j}=\alpha_{0}
$$

By evaluating (31) at any feasible solution where $y_{j}=1$, for all $j \in N$, that is tight for (29), and any tight feasible solution where one arc $k \in J$ is closed and all other arcs are open, we get $-\bar{\alpha}\left(m_{k}-\lambda\right)^{+}-\beta_{k}=0$. Varying over all possible choices of $k$ gives

$$
\beta_{j}=-\bar{\alpha}\left(m_{j}-\lambda\right)^{+} \text {for all } j \in J
$$

Finally we need to determine the value of $\alpha_{0}$. By using the value (32) of $\beta_{j}$ for all $j \in J$ in equation (31), and by evaluating (31) at any feasible point that is tight for (29) we obtain

$$
\alpha_{0}=\bar{\alpha}\left(b-\sum_{j \in J}\left(m_{j}-\lambda\right)^{+}\right)+\gamma b
$$

which completes the first part of our proof.

Necessity: Suppose that $m_{j} \leq \lambda$ for all $j \in J$. Then the flow cover inequality (29) reduces to $\sum_{j \in J} x_{j} \leq b$. This inequality is dominated by the valid inequality $\sum_{j \in N} x_{j} \leq b$ and can therefore not be facet defining.

\subsubsection{Separation based on the flow cover inequalities}

Let $\left(x^{*}, y^{*}\right)$ denote a fractional solution to the LP-relaxation of the single-node capacitated flow problem (26)-(28). Moreover, let $z_{j}, j \in N$ be a zero-one variable that takes value one if $j \in J$, and value zero otherwise. For a given value of $\lambda>0$, the problem of finding the most violated flow cover inequalities (29) is formulated as the following knapsack type problem.

$$
\begin{array}{ll}
\max & \sum_{j \in N}\left[x_{j}^{*}+\left(m_{j}-\lambda\right)^{+}\left(1-y_{j}^{*}\right)\right] z_{j} \\
\text { s.t. } & \sum_{j \in N} m_{j} z_{j}=b+\lambda \\
& z_{j} \in\{0,1\} \quad \text { for all } j \in N
\end{array}
$$

Crowder et al. (1983), and VAN Roy and Wolsey (1987) discuss a heuristic for problem (33)-(35).

(C) VVS, 1999 


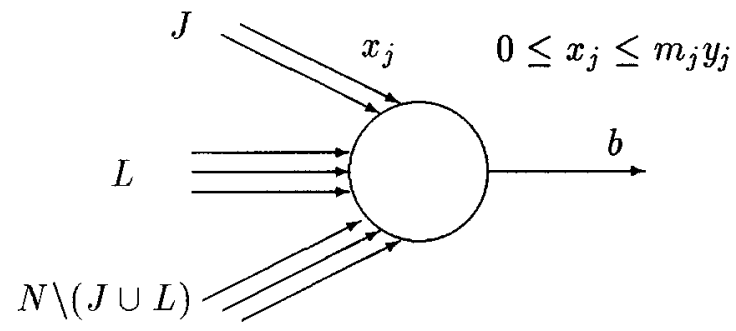

Fig. 7. The single-node capacitated flow model with sets $J, L$ and $N \backslash(J \cup L)$.

\subsubsection{The extended flow cover inequalities}

Once we have a set $J$ satisfying the conditions of Theorem 5 we can extend the flow cover inequality by including flow from the arcs belonging to the set $L \subseteq(N \backslash J)$, see Figure 7.

Let $\bar{m}=\max _{j \in J}\left\{m_{j}\right\}$, and let $\bar{m}_{l}=\max \left\{\bar{m}, m_{l}\right\}$ for all $l \in L$. The following extended flow cover inequality is valid for $\operatorname{conv}\left(X_{F C}\right)$.

$$
\sum_{j \in J \cup L} x_{j} \leq b-\sum_{j \in J}\left(m_{j}-\lambda\right)^{+}\left(1-y_{j}\right)+\sum_{j \in L}\left(\bar{m}_{j}-\lambda\right) y_{j}
$$

It is only interesting to include arc $l$ in the set $L$ if $m_{l}>\bar{m}-\lambda$, since we otherwise obtain a stronger inequality by combining (36) with $L \backslash\{l\}$, with the defining constraint $x_{l} \leq m_{l} y_{l}$. PADBERG et al. (1985) showed that if $0<\bar{m}-\lambda<m_{l} \leq \bar{m}$ for all $l \in L$, then the extended flow cover inequality (36) defines a facet of $\operatorname{conv}\left(X_{F C}\right)$, and obtained the following result in the equal capacity case. Let $X_{F C}^{C}$ denote the set of vectors corresponding to feasible solutions to (26)-(28) if $m_{j}=m$ for all $j \in N$.

Theorem 6 Padberg, VAN Roy, and Wolsey (1985). Assume that $m_{j}=m$ for all $j \in N$, and that $b$ is not an integer multiple of $m$. Let $l=[b / m]$, and $\lambda=m l-b$. Let $S$ be any flow cover with respect to $N$, i.e., $|S| \geq l$. The extended flow cover inequalities

$$
\sum_{j \in S} x_{j} \leq b+\sum_{j \in S}(m-\lambda) y_{j}-(m-\lambda) l
$$

together with the defining inequalities (26), (27) with $m_{j}=m$ for all $j \in N$, and the inequalities $0 \leq y_{j} \leq 1$ for all $j \in N$, define the convex hull of $X_{F C}^{C}$.

The idea behind the proof is as follows. First, the authors characterize the optimal solution to the problem (26)-(28), with $m_{j}=m$ for all $j \in N$, given an arbitrarily chosen objective function. Call this solution $(\hat{x}, \hat{y})$. Next, they show that such an optimal solution can be obtained by solving an assignment problem, which we shall refer to as problem $A P$. The next step is to consider the linear formulation that we assume defines the convex hull of feasible solutions, i.e., the formulation given in the theorem, and its dual. Finally, they characterize a dual feasible solution, given the same arbitrarily chosen primal objective function as above, and show that it has the same value as the optimal solution to the dual of the assignment problem $A P$, and (C) VVS, 1999 
hence to $A P$, which implies that it also has the same value as the optimal solution $(\hat{x}, \hat{y})$.

AARDAL et al. (1995) showed that the separation problem based on the family of extended flow cover inequalities can be solved in polynomial time if $m_{j}=m$ for all $j \in N$.

VAN Roy and Wolsey (1986) also studied the single-node flow model with both fixed charge inflow and outflow arcs. Separation heurstics for these inequalities are also discussed by VAN Roy and WoLSEY (1987).

\subsection{Applications}

\subsubsection{The economic lot-sizing problem}

As seen in the previous subsection it is sometimes possible to describe the convex hull of feasible solutions by concise families of valid inequalities. For some problems, like the bipartite matching problem, these families contain polynomially many inequalities. In general, however, the families contain exponentially many inequalities as for instance the class of extended flow cover inequalities (37). The first complete convex hull descriptions are due to Edmonds, who characterized the matching polytope (1965) and the polymatroid polytope (1970). In this subsection we briefly discuss such proof techniques and give a proof of the complete characterization of the convex hull of feasible solutions to the economic lot-sizing problem.

In the primal method we start by considering an arbitrary valid and facet defining inequality $a x \leq b$. We then determine all relations between the coefficients $(a \mid b)$ of this inequality that can be obtained under the assumptions that the inequality is valid and facet defining. Finally, it is shown that all feasible combinations of $a$ and $b$ precisely yield the facet defining inequalities that were assumed to define the convex hull. This technique usually involves a lot of technicalities, and is therefore used less frequently.

In the dual proof technique we want to prove that a given linear description $P$ defines the convex hull of feasible solutions $X$. We take an arbitrary objective function $c$, and solve the dual problem of $\min \{c x: x \in P=\operatorname{conv}(X)\}$. We then try to identify a primal solution $x \in X$ that satisfies the complementary slackness conditions given $c$. Since there is an objective function for each extreme point $x$ of $P$, such that $x$ is the unique optimum, this proves that each extreme point of $P$ is in $X$, which in turn proves that $P$ is the convex hull of $X$. Lovísz (1983) uses this technique to characterize the convex hull of the polymatroid polytope. The proof by PADBERG et al. (1985) that the extended flow cover inequalities (37) describe the convex hull of the single-node flow model with equal capacities is also of this type, see Section 2.5.3. An elegant variant of the primal technique has been used by Lovísz (1979) on the matching polytope. He considers an arbitrary objective function $c$, and the set $F$ of optimal solutions of $X$ with respect to $c$. It is then shown that $F$ is contained in one of the faces defined by the families of valid inequalities and the defining constraints that are assumed to describe the convex hull. This shows that the assumed description is complete since if we (C) VVS, 1999 
choose an objective function parallel to a facet, then this facet is the only inequality that is satisfied at equality by all points in $F$.

We illustrate the technique of Lovísz (1979) on the economic lot-sizing (ELS) problem. In ELS we are given $T$ time periods constituting the planning horizon. In each period there is a nonnegative demand $d_{t}$ that has to be satisfied with production in one of the periods $\{1, \ldots, t\}$. We have a per unit production cost $c_{t}$ in each period, and a set-up cost, $f_{t}$, that is incurred whenever there is positive production in period $t$. Let $d_{i, j}$ denote the cumulative demand of the periods $\{i, \ldots, j\}$. The standard formulation of ELS involves nonnegative production variables $x_{t}$ and binary set-up variables $y_{t}$.

$$
\begin{aligned}
& \min \sum_{t=1}^{T}\left(f_{t} y_{t}+c_{t} x_{t}\right) \\
& \text { s.t. } \quad \sum_{t=1}^{T} x_{t}=d_{1, T} \\
& \sum_{\tau=1}^{t} x_{\tau} \geq d_{1, t} \quad \text { for all } t=1, \ldots, T-1 \\
& x_{t} \leq d_{t, T} y_{t} \quad \text { for all } t=1, \ldots, T \\
& y_{t} \leq 1 \quad \text { for all } t=1, \ldots, T \\
& x_{t}, y_{t} \geq 0 \quad \text { for all } t=1, \ldots, T \\
& y_{t} \text { integral } \quad \text { for all } t=1, \ldots, T
\end{aligned}
$$

Equation (38) models the restriction that there is no inventory at the beginning and the end of the planning horizon. Constraints (39) ensure that the inventory at the end of each period is nonnegative, and that all demand is met. Finally, inequalities (40) force a set-up in each period that has positive production. We assume that $d_{1}>0$, which implies that the production in period 1 is positive, and thus the corresponding set-up variable $y_{1}=1$. We denote the vectors corresponding to the set of solutions satisfying (38)-(43) by $X_{E L S}$. Due to the equations (38) and $y_{1}=1$, the dimension of $\operatorname{conv}\left(X_{E L S}\right)$ is at most $2 T-2$. In fact, $\operatorname{dim}\left(\operatorname{conv}\left(X_{E L S}\right)\right)$ is precisely equal to $2 T-2$.

In Part I we described the following so-called $(l, S)$-inequalities introduced by BÁRÁNY et al. (1984) for ELS.

$$
\sum_{t \in\{1, \ldots, l\} \backslash S} x_{t}+\sum_{t \in S} d_{t, l} y_{t} \geq d_{1, l} \quad \text { for all } l \in\{1, \ldots, T\}, \text { and all } S \subseteq\{1, \ldots, l\}
$$

The $(l, S)$-inequalities constitute a subfamily of the fixed charge uncapacitated network inequalities (24), cf. Figure 8 . To see the similarity more clearly we rewrite the $(l, S)$-inequalities in the following equivalent form:

$$
\sum_{t \in S} x_{t} \leq \sum_{t \in S} d_{t, l} y_{t}+s_{l} \text { for all } l \in\{1, \ldots, T\}, \text { and all } S \subseteq\{1, \ldots, l\}
$$




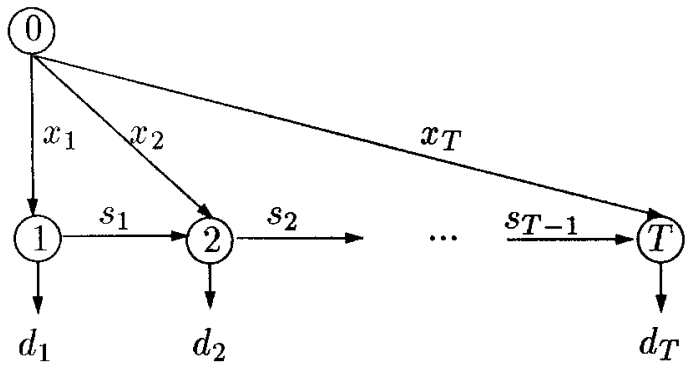

Fig. 8. The economic lot-sizing problem represented as a network.

where $s_{1}=x_{1}-d_{1}$ and $s_{t}=s_{t-1}+x_{t}-d_{t}$, for all $t=2, \ldots, T$. Let $A=\left\{(0, t)_{t=1}^{T},(t\right.$, $\left.t+1)_{t=1}^{T-1}\right\}$, and let $x_{t}$ denote the flow along arcs $(0, t), s_{t}$ de flow along $\operatorname{arcs}(t, t+1)$, and $d_{t}$ the outflow from node $t$. Moreover, let $X=\{1,2, \ldots, l\}, R=\{E(X)\}$ and $C=\left\{\left(0, i_{1}\right), \ldots,\left(0, i_{r}\right)\right\}$, with $i_{j} \in\{1, \ldots, l\}$ for $j=1, \ldots, r$, such that $H=\left\{i_{1}, \ldots\right.$, $\left.i_{r}\right\} \subseteq\left\{1, \ldots, l_{\}}\right.$. We also note that the inequalities (39) and (40) are special cases of the $(l, S$ )-inequalities. Inequalities (39) can be obtained by taking $S=\emptyset$, and inequalities (40) are obtained by taking $S=\{k\}$. Bárány et al. proved that $\operatorname{conv}\left(X_{E L S}\right)$ is defined by the $(l, S)$-inequalities together with the constraints $y_{1}=1,(38),(41)$, and (42). We will prove this result by using Lovász's technique (see Lovász, 1979).

Let $F$ be the set of optimal solutions of $\operatorname{conv}\left(X_{E L S}\right)$ with respect to the objective function $f y+c x$. To simplify the analysis we adjust the objective function in the following way. Let $c_{\min }=\min _{t \in\{1, \ldots, T\}}\left\{c_{t}\right\}$. We first add the constant $c_{\min } d_{1, T}=$ $c_{\min } \Sigma_{t=1}^{T} x_{t}$ to the objective function, and then subtract $c_{\min }$ from every per unit production $\operatorname{cost} c_{t}$. This ensures that the minimum per unit production cost among all periods is zero, and does not change the optimal solution since $\Sigma_{t} x_{t}=d_{1, T}$. Similarly, we add the value $f_{1} y_{1}=f_{1}$ to the objective function, and set $f_{1}$ to zero.

Case 1. Suppose that $f_{t}<0$ for some $t \in\{2, \ldots, T\}$.

Then all solutions in $F$ satisfy $y_{t}=1$, since a solution with $y_{t}=0$ can be improved by setting $y_{t}=1$. Hence, the face defined by $y_{t}=1$ contains $F$.

From now on we may assume that $f_{t} \geq 0$ for all $t \in\{1, \ldots, T\}$. Define $l=\max \left\{t: c_{\tau}>0\right.$ or $f_{\tau}>0$ for all $\left.\tau \leq t\right\}$. Hence, if $l<T$, then $f_{l+1}=c_{l+1}=0$.

Case 2. Suppose that there is a period $t \in\{1+2, \ldots, T\}$ such that $f_{t}>0$.

Then all solutions in $F$ satisfy $y_{t}=0$, since a solution with $y_{t}=1$ can be improved by setting $y_{t}=0$, and, if necessary, moving production in $t$ to period $l+1$ at a cost reduction. Hence, the face defined by $y_{t}=0$ contains $F$.

We deal with periods $t>l+1$ for which $c_{t}>0$ in a similar way by showing that the face defined by $x_{t}=0$ contains $F$. From now on, we may assume that for all $t>l$ we have $f_{t}=c_{t}=0$. Moreover, we may assume that $l \geq 1$, otherwise the objective function has zero coefficients only and $F$ is not a proper face of $\operatorname{conv}(X)$. Define $S=\left\{t \leq l: c_{t}=0\right\}$. 
If $l=T$ then $S$ is not empty, since the minimum unit production cost is zero. Hence, the $(l, S)$-inequality based on $l$ and $S$ as defined above, is not implied by equation (38). Moreover, if $l=1$, then $c_{1}>0$, which ensures that the $(l, S)$-inequality is not implied by $y_{1}=1$ either.

Case 3. We prove that all solutions of $F$ satisfy the $(l, S)$-inequality at equality. Suppose that there is a solution $(\bar{y}, \bar{x})$ in $F$ for which this is not true, i.e.,

$$
\sum_{t \in\{1, \ldots, l\} \backslash S} \bar{x}_{t}+\sum_{t \in S} d_{t, l} \bar{y}_{t}>d_{1, l}
$$

Let $u$ be the smallest index in $S$ with $\bar{y}_{u}=1$, if it exists, otherwise set $u=l+1$. Production that takes place in $\{u+1, \ldots, l\}$ can be moved to $u$ at a cost reduction, since these periods have positive unit production costs, or positive set-up costs. Therefore, all production in $\{u, \ldots, l\}$ takes place in $u$. It follows from (46) that there must be overproduction in the periods $\{1, \ldots, u-1\} \backslash S$, which can be moved to $u$ at a further cost reduction. Hence, we can conclude that any solution satisfying (46) is not cost optimal. This finishes the proof.

In VAN HoEsel et al. (1994) a similar proof is given for the more general economic lot-sizing problem with additional start-up costs.

\subsubsection{The facility location problem}

Here we shall discuss how odd-hole inequalities and flow cover inequalities can be used, and extended, when solving facility location problems. The facility location problem is defined as follows. Let $M=\{1, \ldots, m\}$ be the set of facilities, and let $N=\{1, \ldots, n\}$ be the set of clients. Facility $j$ has capacity $m_{j}$, and client $k$ has demand $d_{k}$. The total demand of the clients in the set $S \subseteq N$ is denoted by $d(S)$. The fixed cost of opening facility $j$ is equal to $f_{j}$ and the cost of transporting one unit of goods from facility $j$ to client $k$ is equal to $c_{j k}$. Let $y_{j}=1$ if facility $j$ is open and let $y_{j}=0$ otherwise. The flow from facility $j$ to client $k$ is denoted by $v_{j k}$. We want to determine which facility should be opened and how the flow should be distributed between the open facilities and the clients such that the sum of the fixed costs of opening the facilities, and the transportation costs is minimized, and such that all clients are served, and all capacity restrictions are satisfied. The mathematical formulation is given below

$$
\begin{array}{lll}
\min & \sum_{j \in M} f_{j} y_{j}+\sum_{j \in M} \sum_{k \in N} c_{j k} v_{j k} & \\
\text { s.t. } & \sum_{j \in M} v_{j k}=d_{k} & \\
& \sum_{k \in N} v_{j k} \leq m_{j} y_{j} & \text { for all } k \in N \\
& 0 \leq v_{j k} \leq d_{k} y_{j} & \text { for all } j \in M \\
& y_{j} \in\{0,1\} & \text { for all } j \in M, k \in N
\end{array}
$$


Inequalities (49) are redundant with respect to the integer formulation, but they do strengthen the linear programming relaxation of the facility location problem quite substantially.

\section{The Uncapacitated Case}

In the uncapacitated facility location (UFL) problem we have $m_{j} \geq d(N)$ for all $j \in M$. It is convenient to scale the flow by substituting the variables $v_{j k}$ by the variables $x_{j k}=v_{j k} / d_{k}$. The set of feasible solutions to UFL, $X_{U F L}$, is given by the following sets of constraints.

$$
\begin{array}{ll}
\sum_{j \in M} x_{j k}=1 & \text { for all } k \in N \\
0 \leq x_{j k} \leq y_{j} & \text { for all } j \in M, k \in N \\
y_{j} \in\{0,1\} & \text { for all } j \in M
\end{array}
$$

It is possible to require explicitly that $x_{j k} \in\{0,1\}$ since there is at least one optimal solution of UFL having this property. Moreover, we can change the equality sign in constraint set (51) to a less-than-or-equal-to sign if we make an appropriate change in the objective function (for more details see CHO et al., 1983a). Finally, by complementing the $y_{j}$-variables, i.e., by introducing $y_{j}^{\prime}=1-y_{j}$, we obtain the following vertex packing formulation of UFL.

$$
\begin{array}{ll}
\sum_{j \in M} x_{j k} \leq 1 & \text { for all } k \in N \\
x_{j k}+y_{j}^{\prime} \leq 1 & \text { for all } j \in M, k \in N \\
y_{j}^{\prime}, x_{j k} \in\{0,1\} & \text { for all } j \in M, k \in N
\end{array}
$$

Let $X_{U F L V P}$ be the set of feasible solutions to (54)-(56). Given a vertex packing formulation of UFL, we can construct an associated undirected graph, called the intersection graph by introducing a vertex for every variable, and an edge for every pair of nonorthogonal columns, see Figure 9. To determine conv $\left(X_{U F L V P}\right)$ is equivalent to determining the convex hull of vertex packings in the associated intersection graph. Hence, we can use all results described in Section 2.2 to derive valid inequalities for UFL. Due to the construction of the intersection graph all cliques in this graph are described by inequalities (54) and (55), and all odd holes are cycles where every third vertex is a $y_{j}^{\prime}$-vertex, see Figure 10. Both CoRnUÉJOLs and THIzY (1982) and CHo et al. (1983a,b) used the result by Chvatál given in Theorem 1 to find more general inequalities than the odd-hole inequalities. All these inequalities have a regular cyclic structure and all coefficients are equal to one for all variables except one example of a simultaneously lifted odd-hole inequality given by Cornuéjols and Thizy. This lifted odd-hole inequality is precisely the inequality illustrated in Figure 3 in Section 2.2. Aardal and Van Hoesel (1998) discuss further use of simultaneous lifting to get new facets having different coefficients. 


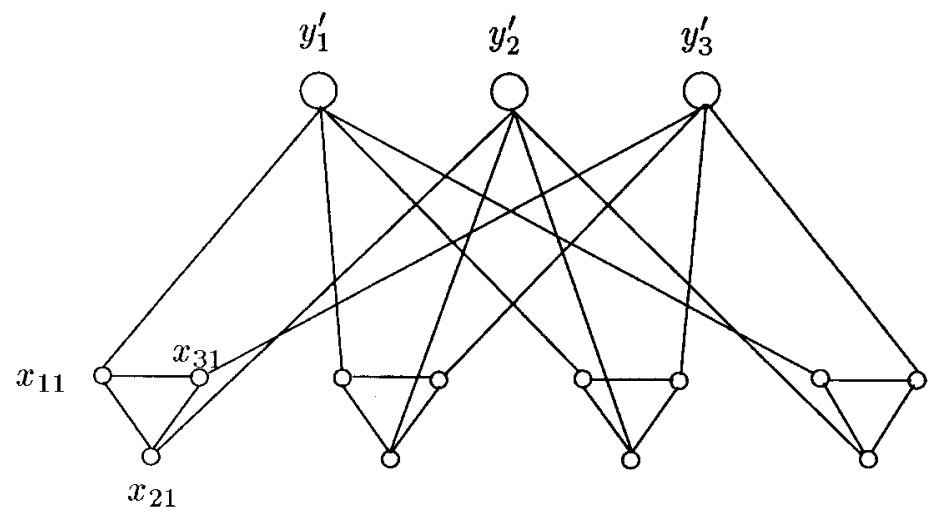

Fig. 9. The intersection graph for $|M|=3$, and $|N|=4$.

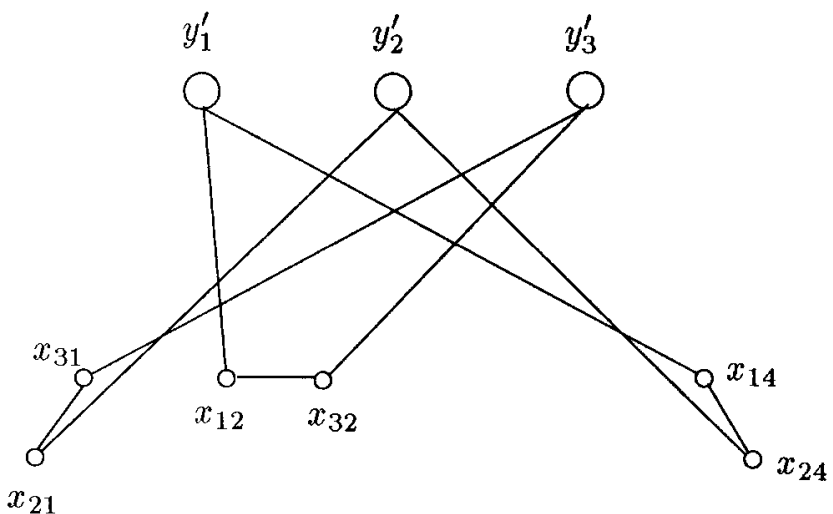

Fig. 10. An odd hole in the intersection graph.

\section{The Capacitated Case}

Here we show how flow cover inequalities can be generated based on aggregate information from the formulation.

Let $v_{j}=\Sigma_{k \in N} v_{j k}$, and consider the following valid, but redundant, constraints.

$$
\begin{aligned}
& 0 \leq v_{j} \leq m_{j} y_{j} \quad \text { for all } j \in M \\
& \sum_{j \in M} v_{j}=d(N)
\end{aligned}
$$

In Section 2.3 of Part I of this article, we described how we can combine constraints (57) and (58) with constraints (50) to obtain the knapsack polytope $X_{K}^{C F L}=\left\{y^{\prime} \in\{0\right.$, $\left.1\}^{|M|}: \sum_{j \in M} m_{j} y_{j}^{\prime} \leq \Sigma_{j \in M} m_{j}-d(N)\right\}$, where $y_{j}^{\prime}=1-y_{j}$. Knowing that the knapsack polytope $X_{K}^{C F L}$ forms a relaxation of the capacitated facility location (CFL) problem, we can conclude that the knapsack cover inequalities generated from $X_{K}^{C F L}$ are valid for CFL. If we again combine the aggregate constraints with constraint (50) we can obtain the single-node flow polytope $\left\{(v, y) \in \mathbb{R}^{|M|} \times\{0,1\}^{|M|}: \Sigma_{j \in M} v_{j}=d(N)\right.$, (c) VVS, 1999 


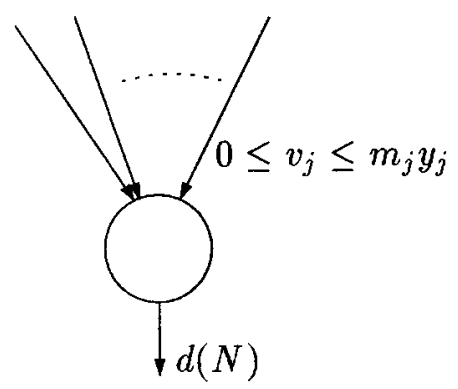

Fig. 11. The single-node flow relaxation of CFL.

$0 \leq v_{j} \leq m_{j} y_{j}, j \in M$ \}, see Figure 11. Hence we can use the flow cover inequalities when solving CFL. The first step in generalizing the flow cover inequality is made by considering inequalities based on subsets $K \subseteq N$ of clients. One way of generalizing the flow cover inequalities further is by considering a subset of clients as well as subsets of arcs yielding the family of effective capacity inequalities (AARDAL et al., 1995). Let $K_{j} \subseteq K$ for all $j \in M$ and let $\bar{m}_{j}=\min \left\{m_{j}, d\left(K_{j}\right)\right\}$. Let $J$ define a flow cover with respect to $K$, i.e., $\Sigma_{j \in J} \bar{m}_{j}=d(K)+\lambda$ with $\lambda>0$. The effective capacity (EC) inequality

$$
\sum_{j \in J} \sum_{k \in K_{j}} v_{j k} \leq d(K)-\sum_{j \in J}\left(\bar{m}_{j}-\lambda\right)^{+}\left(1-y_{j}\right)
$$

is valid for $\operatorname{conv}\left(X_{C F L}\right)$. The facet defining EC inequalities were completely characterized by AARDAL et al. (1995).

Example 4 Consider the CFL structure given in Figure 12. Let $J=\{1,2,3\}, K=\{1$, $2,3,4\}, K_{1}=\{3,4\}, K_{2}=\{1\}$, and $K_{3}=K$. We have $\lambda=\Sigma_{j \in J} \bar{m}_{j}-d(K)=9$.

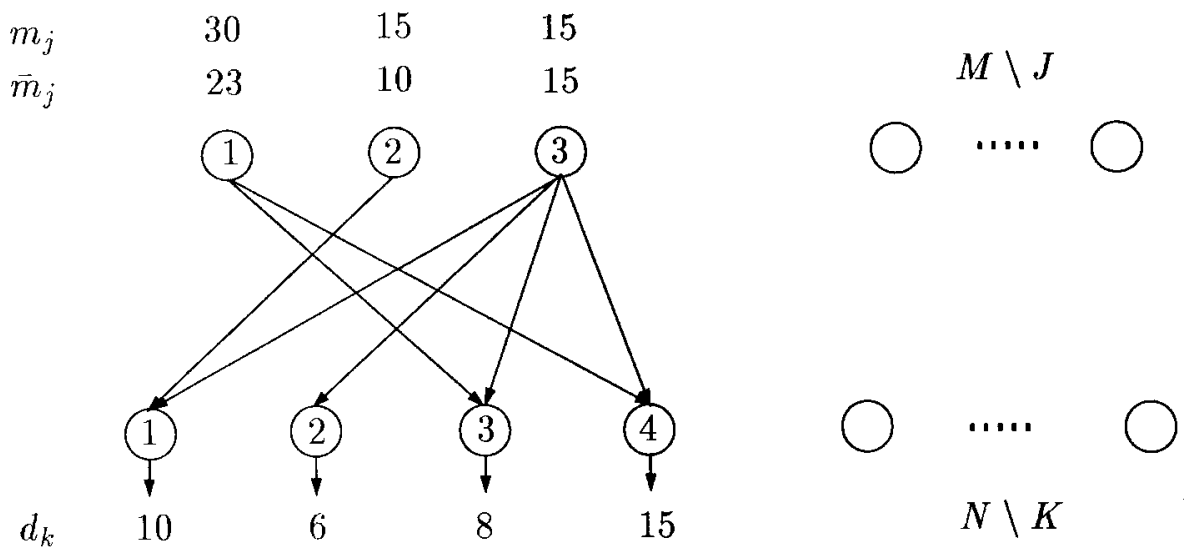

Fig. 12. A CFL structure. 
The facet defining EC inequality based on this structure is

$$
\begin{aligned}
& v_{13}+v_{14}+v_{21}+v_{31}+v_{32}+v_{33}+v_{34} \\
& \quad \leq 39-14\left(1-y_{1}\right)-\left(1-y_{2}\right)-6\left(1-y_{3}\right) .
\end{aligned}
$$

A further generalization of the flow cover inequalities, called the family of submodular inequalities, was developed by WoLSEY (1989) and adapted to the capacitated facility location problem by AARDAL et al. (1995). The separation problem based on the EC inequalities and the submodular inequalities, as well as computational experience from using these inequalities, are discussed by AARDAL (1998).

\subsection{A list of polyhedral results for combinatorial problems}

Here we provide a list of polyhedral results that are known for combinatorial optimization problems. If a recent survey of results for a specific problem class is known, we refer to the survey and not to the individual articles. Surveys are marked with an asterisk. Due to the vast literature, we do not claim the list to be complete. Airline fleet and crew scheduling: Hoffman and PADBerg (1993), Hane et al. (1995). Boolean quadratic polytope: Padberg (1989), Lee and Leung (1993a). Clique problems: Grötschel and WakabaYashi (1989, 1990). Coloring: Lee and Leung (1993b), Nemhauser and Park (1991). Covering, packing and partition: Balas and Padberg (1972), Padberg (1973, 1977, 1980), Nemhauser and Trotter (1974), Trotter (1975), Wolsey (1976b), Balas and Zemel (1977), Balas and Ho (1980), Balas and NG (1989a,b), Cornuésols and Sassano (1989), Laurent (1989), Nobili and Sassano (1989), SAssano (1989), Chopra and Rao (1993), Ferreira et al. (1996, 1998), Müller and Schulz (1996), Cheng and Cunningham (1997). Cut polytopes: Barahona and Mahjoub (1986), Barahona et al. (1988), Conforti et al. (1990/ 91a,b), De Sousa and Laurent (1995), Deza et al. (1992), Deza and Laurent (1992a,b), Pulleyblank and Shepherd (1993), Balas et al. (1994b), Brunetta et al. (1997). Frequency assignment: AARDAL et al. (1995). General integer and mixed 0-1 structures: Wolsey (1976a), Peled (1977), Zemel (1978), Crowder et al. (1983), Padberg et al. (1985), Van Roy and Wolsey (1985, 1986, 1987), Goemans (1989), Nemhauser and Wolsey (1990), Caprara and Fischetti (1996), Balas et al. (1996a,b), Ceria et al. (1998). Knapsack problems: Balas (1975), Hammer et al. (1975), Wolsey (1975), Balas and Zemel (1978), Padberg (1980), Nemhauser and Vance (1994), Gu et al. (1995), Weismantel (1997), Pochet and Weismantel (1998). Layout design: Leung (1994). Linear ordering: Grötschel et al. (1984, 1985b), Reinelt (1985), Mitchell and Borchers (1992, 1993). Location: Cornú́sols et al. (1977), Cornuésols and Thizy (1982), Cho et al. (1983a,b), Leung and Magnanti (1989), Aardal et al. (1995, 1996), Aardal (1998), Aardal and Van Hoesel (1998). Lot sizing: Pochet and Wolsey (1995)*, Constantino (1998). Matching: Edmonds (1965), Grötschel and Holland (1985). Network and VLSI design: Pochet and Wolsey (1992), Grötschel et al. (1992, 1993, 1995, 1997), BIENSTOCK and GüNLÜK (C) VVS, 1999 
(1996), Bienstock et al. (1998). Postman problems: Grötschel and WiN (1992). Scheduling: Queyranne and Schulz (1994)*. Subgraph polytopes: Balas and Pulleyblank (1983), Barahona et al. (1985), Grötschel et al. (1985a), Jünger (1985), Barahona and Mahjoub (1989, 1992), Chopra (1992), Jünger and Mutzel (1993), Leung and Lee (1994), Goemans and Hall (1996), Bauer (1997). Tenary problems: CHOPRA (1989a). Traveling salesman problems: DANTZIG et al. (1954, 1959), Grötschel and Padberg (1979), Crowder and Padberg (1980), Grötschel (1980), Padberg and Hong (1980), Cornuéjols and Pulleyblank (1982), Grötschel and Pulleyblank (1986), Padberg and Rinaldi (1987, 1990, 1991), Fischetti (1991a, 1992), Grötschel and Holland (1991), NAdDEF and Rinaldi (1991, 1992), Reinelt (1991), Naddef (1992), Clochard and Naddef (1993), Applegate et al. (1994), Balas et al. (1995), Goemans (1995), Fleisher and Tardos (1996), Carr (1997). Trees, forests and arborescences: Gamble and Pulleyblank (1989), Chopra (1989b), Fischetti (1991b), Balas and Fischetti (1992), Chopra et al. (1992), Goemans (1992), Hall and Magnanti (1992), Chopra and Rao (1994a,b), Grötschel et al. (1996), Hall (1996). Vehicle routing: Araque (1989, 1990), Araque et al. (1990), CORNuÉJOls and HARCHE (1993).

\section{Computational aspects}

Once specific classes of valid inequalities for a certain version of ILP (1) have been developed we can implement the separation algorithms for these inequalities in the following cutting plane algorithm, see Figure 13.

\section{Outline of the cutting plane algorithm.}

1. Initialize the linear programming relaxation LP of ILP.

2. Solve LP and let $x^{*}$ be the optimal solution. If $x^{*}$ is integral, stop, otherwise go to step 3 .

3. Separation algorithms are run to identify inequalities violated by $x^{*}$. If one or more inequalities, or cuts, have been found add them to LP and go to step 2. If no violated inequality is found, stop.

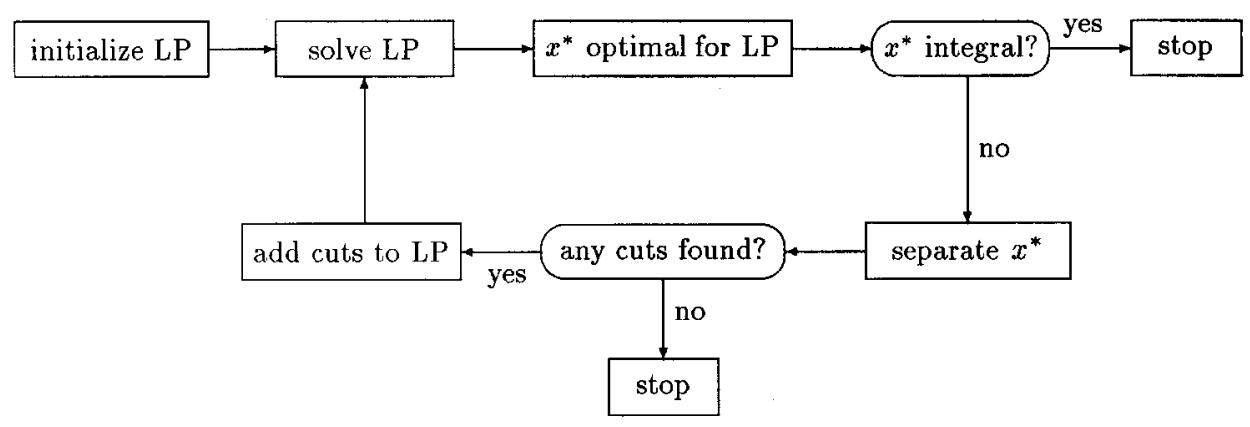

Fig. 13. Basic cutting plane algorithm. 
If the algorithm terminates by finding an integral solution $x^{*}$, then $x^{*}$ is provably optimal. Otherwise, the final fractional solution provides a lower bound on the optimal value, if we assume that ILP is a minimization problem. Contrary to Gomory's cutting plane algorithm, (Gomory, 1958, see Part I) we cannot guarantee that the algorithm terminates with the optimal solution to ILP since we in general consider only a subset of all classes of facet defining inequalities, and since the separation problems are often solved heuristically. Nevertheless, the cutting plane technique has proved very effective for finding at least very strong lower bounds. A good lower bound decreases the expected size of a branch-and-bound tree if we need to obtain the optimal solution. To illustrate how the lower bound develops if we add valid inequalities sequentially, we consider a TSP instance of 120 cities from GrötsCHEL (1980), which was solved to optimality after adding cutting planes only. The optimal solution was found after 13 calls to the LP-solver. The value of the LP relaxation, $z_{L P}$, and the number of added cuts at each iteration, are given in Table 1.

In the remainder of this section we shall discuss how the basic cutting plane algorithm can be extended and embedded in a branch-and-bound framework. We also discuss several implementation issues. Each extension is illustrated by an example or by computational results. In the tables we use the following notation: $z_{L P}$ denotes the value of the LP-relaxation, and $z_{I P}$ and $z_{M I P}$ denote the optimal value of the integer and the mixed-integer optimization problems respectively. By $\%$ gap we mean the percentage duality gap, $100\left(z_{I P}-z_{L P}\right) / z_{I P}$. The percentage duality gap closed, denoted $\%$ gap closed is calculated as $100\left(z_{L P}^{\text {root }}-z_{L P}\right) /\left(z_{I P}-z_{L P}\right)$, where $z_{L P}^{\text {root }}$ is the value of the LP-relaxation after all violated inequalities that have been identified in the root node of the branch-and-bound tree have been added. The number of branch-and-bound nodes needed to verify the optimum is given in the column $B \& B$ nodes.

Table 1. A cutting plane algorithm applied to a 120-city TSP

\begin{tabular}{lll}
\hline Iteration & $z_{L P}$ & Inequalities \\
\hline 1 & 6662.5 & 13 \\
2 & 6883.5 & 15 \\
3 & 6912.5 & 7 \\
4 & 6918.8 & 9 \\
5 & 6928.0 & 6 \\
6 & 6935.3 & 9 \\
7 & 6937.2 & 8 \\
8 & 6939.5 & 5 \\
9 & 6940.4 & 4 \\
10 & 6940.8 & 12 \\
11 & 6941.2 & 5 \\
12 & 6941.5 & 3 \\
13 & 6942.0 & \\
\hline
\end{tabular}




\subsection{Extending the cutting plane algorithm}

There are several ways to extend the basic cutting plane algorithm. We will describe the major additional techniques in the order in which they appear in an extended cutting plane algorithm.

\subsubsection{Preprocessing}

Preprocessing integer linear programs involves removing redundant constraints, tightening the constraint coefficients and right-hand sides of the constraints, and fixing variables to certain values. This typically yields better lower bounds provided by the linear relaxation, or a significant reduction in the size of the formulation, both with respect to the number of constraints and number of variables. An important factor is also that the instance becomes numerically more tractable if large coefficients are reduced. There are many preprocessing techniques described in the literature. For each technique, or combination of techniques, one needs to find the right balance between effectiveness and computing time. Here we shall present some simple methods that strengthen a linear program quickly. These methods are described by SAVELSBERGH (1994), and originally developed by Crowder et al. (1983) and Hoffman and PADBERG (1991).

Consider the following subset of constraints from a mixed integer program, where $N^{+}$is the subset of variables with positive coefficients, $N^{-}$is the subset of variables with negative coefficients, and $N=N^{+} \cup N^{-}$. Note that this implies that $a_{j} \geq 0$ for all $j \in N$ in inequality (60) below.

$$
\begin{aligned}
& \sum_{j \in N^{+}} a_{j} x_{j}-\sum_{j \in N^{-}} a_{j} x_{j} \leq b \\
& l_{j} \leq x_{j} \leq u_{j} \quad \text { for all } j \in N
\end{aligned}
$$

A lower bound on the left-hand side of (60) is $L B=\Sigma_{j \in N^{+}} a_{j} l_{j}-\Sigma_{j \in N^{-}} a_{j} u_{j}$. If $L B>b$, then the problem is infeasible. An upper bound on the left-hand side of (60) is $U B=\sum_{j \in N^{+}} a_{j} u_{j}-\sum_{j \in N^{-}} a_{j} l_{j}$. If $U B \leq b$, then the constraint is redundant. It is also possible to tighten the bounds (61) on the variables by considering one variable at the time. Consider variable $x_{k}, k \in N^{+}$, and let $L B_{k}=\sum_{j \in N^{+} \backslash\{k\}} a_{j} l_{j}-\sum_{j \in N^{-}} a_{j} u_{j}$. Clearly, every feasible solution satisfies $x_{k} \leq\left(b-L B_{k}\right) / a_{k}$. Hence, the upper bound $u_{k}$ can be decreased if $u_{k}>\left(b-L B_{k}\right) / a_{k}$. Analogous results can be obtained for the lower bound $l_{k}$, and for the upper and lower bounds of variables $x_{k}$ where $k \in N^{-}$.

An elegant preprocessing technique is "probing" on the variables, which means fixing variables temporarily. Probing techniques were introduced by GuIGNARD and SPielberg (1981). By fixing a variable we may detect logical relations between variables that can be used to tighten, and reduce the size of the formulation as is demonstrated in the following example. Consider the following set of (c) VVS, 1999 
constraints with two nonnegative real variables $x_{1}$ and $x_{2}$ and two binary variables $y_{1}$ and $y_{2}$.

$$
\begin{aligned}
x_{1}+3 x_{2} & \geq 12 \\
2 x_{1}+x_{2} & \geq 15 \\
x_{1} & \leq 10 y_{1} \\
x_{2} & \leq 20 y_{2}
\end{aligned}
$$

We probe on $y_{1}$ by setting $y_{1}$ equal to zero. Then, by the third constraint, $x_{1}$ has to be equal to zero as well, which, due to the second and fourth constraints, implies that $x_{2} \geq 15$ and $y_{2}=1$. If we consider the first constraint we see that if $y_{1}=0$, then we can increase the right-hand side to 45 . If however $y_{1}=1$ then the right-hand side has to be equal to 12 . Hence, it is possible to add the term $(45-12)\left(1-y_{1}\right)$ to the righthand side of the first constraint that now becomes

$$
x_{1}+3 x_{2} \geq 12+33\left(1-y_{1}\right)
$$

Implication inequalities derived from binary variables can also be used to obtain clique constraints. In the previous example we saw that $y_{1}=0$ implies $y_{2}=1$. Thus, we have $y_{1}^{\prime}+y_{2}^{\prime} \leq 1$, where $y_{i}^{\prime}, i=1,2$ denotes the complement of the variable of $y_{i}$. To find such clique inequalities we can construct an auxiliary graph that has one vertex for every variable and its complement. Two vertices are connected by an edge if the corresponding variables cannot both have value one. Consider the auxiliary graph shown in Figure 14. From the structure of the graph we conclude that $y_{2}^{\prime}$ has to be equal to zero. To see that this is true note that $y_{2}^{\prime}=1$ implies $y_{2}=0$. If $y_{2}=0$, then either $y_{3}=0$ or $y_{3}=1$. If $y_{3}=0$, then $y_{3}^{\prime}=1$, which implies $y_{1}^{\prime}=0$, which in turn implies that $y_{1}=1$. This is however not feasible since $y_{1}$ is adjacent to $y_{2}^{\prime}$. A similar contradiction is obtained if we choose $y_{3}=1$. This example shows that by investigating logical implication we may be able to fix variables and thereby reduce the problem size. Moreover, the cliques in the auxiliary graph do in general induce inequalities that are stronger than the inequalities in the original formulation.

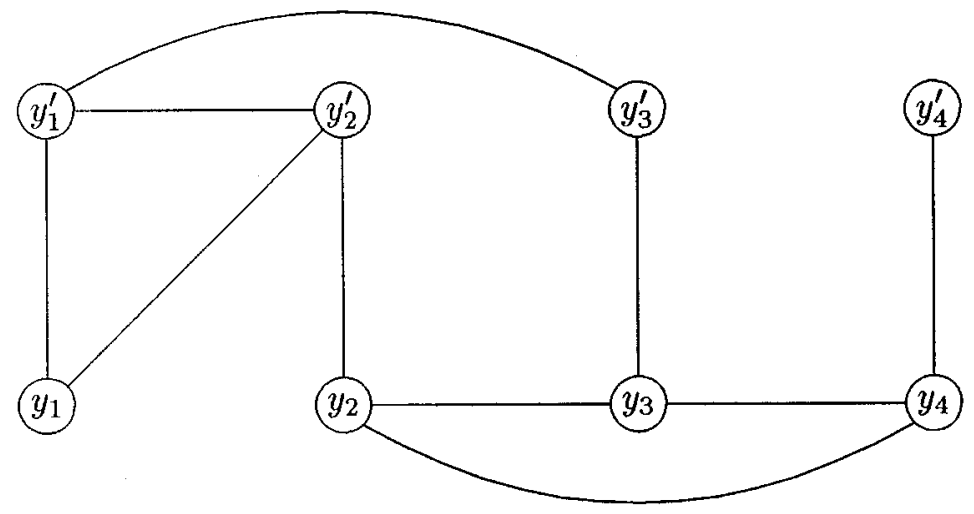

Fig. 14. Auxiliary vertex packing graph.

(C) VVS, 1999 
Table 2. Effect of preprocessing technqiues

\begin{tabular}{|c|c|c|c|c|c|}
\hline Problem & $\begin{array}{l}z_{L P} \text { without } \\
\text { prepr. }\end{array}$ & $\begin{array}{l}z_{L P} \text { with } \\
\text { prepr. }\end{array}$ & $z_{M I P}$ & $\begin{array}{l}\text { B\&B nodes } \\
\text { without prepr. }\end{array}$ & $\begin{array}{l}\text { B\&B nodes } \\
\text { with prepr. }\end{array}$ \\
\hline egout & 149.5 & 562.1 & 568.1 & 553 & 3 \\
\hline fixnet3 & $40,717.0$ & $50,414.2$ & $51,973.0$ & 131 & 5 \\
\hline fixnet4 & 4257.9 & 7703.4 & 8936.0 & 2561 & 1031 \\
\hline fixnet6 & 1200.8 & 3192.5 & 3983.0 & 4795 & 4305 \\
\hline khb05250 & $95,919,464.0$ & $106,750,366.0$ & $106,940,226.0$ & 11,483 & 13 \\
\hline gen & $112,130.0$ & $112,271.0$ & $112,313.0$ & 11 & 15 \\
\hline att & 125.9 & 149.1 & 160.2 & 6459 & 127 \\
\hline sample2 & 247.0 & 290.4 & 375.0 & 336 & 51 \\
\hline p0033 & 2520.8 & 2838.5 & 3089.0 & 15 & 7 \\
\hline lseu & 834.6 & 947.9 & 1120.0 & 297 & 464 \\
\hline
\end{tabular}

The effectiveness of the various preprocessing techniques has been tested by SAVELSBERGH (1994) on a set of 10 mixed integer programming problems from the literature. Table 2 shows the improvement of the lower bound after preprocessing as well as the reduction in the number of branch-and-bound nodes needed to verify the optimal solution. Observe that the linear programming bound increases substantially for all problems, and that the size of the branch-and-bound tree decreases by a fair amount for most instances. For two instances however, the number of branch-andbound nodes of the preprocessed problem is larger than for the original problem. This phenomenon has to do with the relative lack of robustness of branch-and-bound, and is not really well understood. For more details regarding preprocessing we refer to Crowder et al. (1983), Hoffman and Padberg (1991) and Dietrich and Escudero (1990).

\subsubsection{Postprocessing the linear program}

After the linear program is solved, either the optimal solution is found, or, more usually, a fractional solution $x^{*}$ is obtained, which provides a lower bound $z_{L P}$ on the optimal value $z_{I P}$. Suppose we know a feasible solution with value $z_{F}$. The value $z_{F}$ is an upper bound on $z_{I P}$, thus $z_{I P}$ is guaranteed to lie in the interval $\left[z_{L P}, z_{F}\right]$. Heuristics that use the fractional solution $x^{*}$ to create a feasible solution are known as primal rounding heuristics. Besides providing a worst case distance between the lower bound and the optimal value, an upper bound can also be used to fix variables by reduced cost fixing, or more involved, by parametric analysis on a single variable.

\subsubsection{Generating generic inequalities}

Besides the problem specific classes of valid inequalities, we can try to find violated generic inequalities. Many capacitated problems contain knapsack type constraints, in which case we may try to find violated lifted knapsack cover inequalities (18). Other generic classes of valid inequalities are clique inequalities (4), obtained from the auxiliary graph of the binary variables as shown in Figure 14, odd-hole inequalities (5), network inequalities (24), and extended flow cover inequalities (36). The (C) VVS, 1999 
Table 3. Result of adding lifted knapsack cover inequalities to CFL

\begin{tabular}{rlrrrrrr}
\hline Problem & \% gap & $\begin{array}{l}\text { B\&B } \\
\text { nodes }\end{array}$ & Time (s) & $\begin{array}{l}\text { Cover } \\
\text { ineq. }\end{array}$ & $\begin{array}{l}\text { \% gap } \\
\text { closed }\end{array}$ & $\begin{array}{l}\text { B\&B } \\
\text { nodes }\end{array}$ & Time (s) \\
\hline 50331 & 1.5 & 399 & 686 & 13 & 86.0 & 31 & 125 \\
50332 & 1.2 & 691 & 1560 & 58 & 54.3 & 51 & 450 \\
50333 & 1.5 & 259 & 556 & 122 & 54.1 & 89 & 769 \\
50334 & 0.7 & 239 & 493 & 42 & 76.6 & 23 & 213 \\
50335 & 1.3 & 685 & 1232 & 25 & 78.3 & 49 & 248 \\
100751 & 0.7 & 4077 & 22,977 & 295 & 40.9 & 611 & 10,560 \\
100752 & 0.6 & 15,419 & 74,351 & 648 & 55.4 & 1243 & 20,055 \\
100753 & 0.1 & 183 & 761 & 48 & 12.5 & 59 & 844 \\
100754 & 0.3 & 6687 & 40,604 & 228 & 9.1 & 537 & 11,076 \\
100755 & 0.1 & 117 & 621 & 9 & 34.6 & 23 & 406 \\
\hline
\end{tabular}

capacitated facility location problem provides a good insight in what these generic inequalities might offer. Table 3 shows the improvement obtained by adding lifted cover inequalities to the formulation given in Section 2.6.2. The first five instances have 33 facilities and 50 clients, whereas the last five instances have 75 facilities and 100 clients. For more details, see AARDAL (1988).

\subsection{Embedding the cutting plane algorithm in a branch and bound framework}

\subsubsection{The algorithm}

In the early days of polyhedral techniques problems were solved by applying a cutting plane algorithm in the root node of the branch-and-bound tree only, since the LPsolvers were not designed to handle row management in a practical way, which made the implementation quite involved. In the mid-eighties Grötschel et al. (1984) used a cutting plane algorithm in every node of the branch-and-bound tree to solve the linear ordering problem. PADBERG and RiNALDI (1987) called this idea branch-and-cut.

\section{Outline of the branch-and-cut algorithm.}

1. Initialize a list $L$ of subproblems of the original problem. Repeat steps 2 and 3 , until $L$ is empty.

2. Select a subproblem $S$ from $L$.

3. Consider the linear relaxation of $S$ and apply a cutting plane algorithm to the relaxation. If $S$ is not solved, create new subproblems by branching. Put the new subproblems in $L$.

Every subproblem in $L$ corresponds to a node in the branch-and-cut tree. The subproblems that still need to be investigated are called active. In order to avoid complete enumeration the search tree is pruned at subproblem $j$, i.e., no further subproblems are created at node $j$, if one of the following conditions hold: (i) subproblem $j$ is infeasible, (ii) the optimal solution to the linear relaxation of subproblem $j$ is integral, or (iii) $z_{L P}^{j} \geq \bar{z}$, where $\bar{z}$ is the best known upper bound.

In the branch-and-cut algorithm we need to specify a search strategy and a branching strategy, i.e., how to select a subproblem from the list $L$, and how to create new (C) Vvs, 1999 


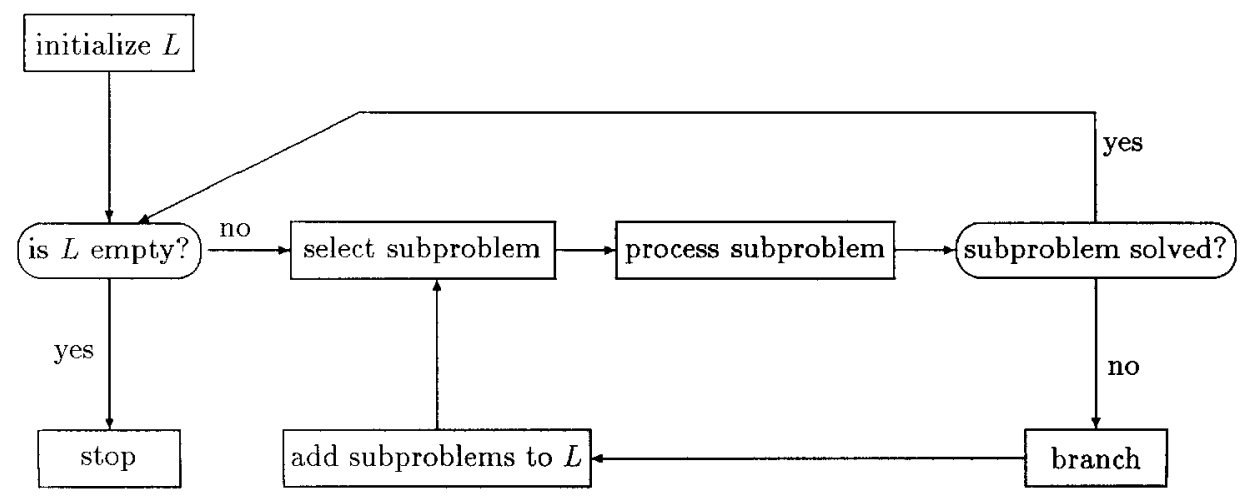

Fig. 15. Branch-and-cut algorithm.

subproblems. The most commonly used search strategies are depth-first search and breadth-first search. In depth-first search one of the subproblems created at the current node is investigated if the current node is not pruned, whereas in breadth-first search all nodes at the current level of the tree are investigated before any node at the level below. The most frequently used branching rules are to branch on a variable according to one, or a mix, of the following four criteria. Here we assume that the variables are binary.

1. Select the variable with value closest to 0.5 .

2. Select the variable with value closest to 1 .

3. Select the variable with highest objective coefficient.

4. Select a set $P$ of "promising" variables and compute for each variable in $P$ the lower bound that is obtained at the corresponding subproblem. Select the variable that yields the smallest lower bound.

PADBerg and RinAldi (1991) suggest a combination of 1 and 3 for the traveling salesman problem. Rule 2 is surprisingly effective in combination with a depth-first strategy. Rule 4, introduced by ApPlEgate et al. (1994), has similarities with the "steepest-edge" idea used in the simplex method for linear programming when choosing the variable to enter the basis. Other strategies have been proposed by BALAS and Tотн (1985). JÜNGER et al. (1992) report on computational experience with various combinations of these rules. When branching on a constraint, usually a clique constraint, a new branch is created for each value that the left-hand side of the constraint can obtain. Clochard and NADDEF (1993) suggest such a rule for the traveling salesman problem.

\subsubsection{Implementation issues}

The various components of the extended cutting plane algorithm may not be very effective in each node of the branch-and-cut tree. Preprocessing for instance has much effect in the root node of the tree since the original formulation of a problem is usually weak at the same time as it contains a lot of redundancy. Similarly, it may be hard to (C) VVS, 1999 
find effective cutting planes in the subproblems further down in the tree. Hence, the major effort on separation is usually put in the root node. In an implementation of a branch-and-cut algorithm we can therefore introduce selection mechanisms indicating where in the tree certain components should be performed. Effectiveness versus computational effort should then be weighed against each other.

As mentioned above, the search tree can be pruned at a certain node if the lower bound obtained at that node exceeds the best known upper bound. In order to decrease the expected size of the search tree it is therefore crucial to compute a good upper bound by a primal heuristic before entering the branching phase.

Branch pausing, introduced by PADBERG and RINALDI (1991), is a strategy where subproblems are temporarily ignored if the lower bounds are greater than a certain threshold value. The threshold value is an estimate of the optimal value of the problem. The advantage with branch pausing is that the expected size of the search tree gets smaller. If we choose to consider subproblems in the order of increasing value of the lower bounds the implementation however gets quite complicated since subsequently chosen subproblems are not necessarily related.

Maintaining the cutting planes is a rather difficult implementation issue. In early versions of branch-and-cut packages, one was only allowed to generate globally valid inequalities, i.e., inequalities that are valid for the original problem instance. These inequalities were maintained in a central pool, from which one could select violated inequalities for the current subproblem. The global cuts usually work well, but to use the full power of the branch-and-cut algorithm, one should also be able to generate inequalities that are locally valid. BALAS et al. (1996) report on good results using branch-and-cut with locally valid Gomory cuts. When solving large instances it becomes important to work with a formulation that is as small as possible. One important feature is therefore to be able to delete inequalities from the active formulation and store them in a pool. A detailed overview of general implementation ideas can be found in JÜNGER et al. (1995). Data structures and other implementation details specific for the traveling salesman problem can be found in APPLEGATE et al. (1994). To conclude this section we show in Figure 16 the branch-and-cut tree of a 532-city traveling salesman problem solved by PADBERG and RINALDI (1987). This tree gives an indication of the development of the lower bound at different levels of the tree. At the first node we give the starting LP-value, and at the second node we give the LP-value after all cuts generated in the root node have been added. Note that after the second level of the tree all values are of the order 27,000 , so we only give the digits as of the hundreds.

\section{Computational results for selected problems}

To give an idea of how polyhedral techniques perform, and how large instances can be solved, we have selected a number of problem types for which computational results are reported in the literature. For a more extensive survey we refer to JÜNGER et al. (1995). 


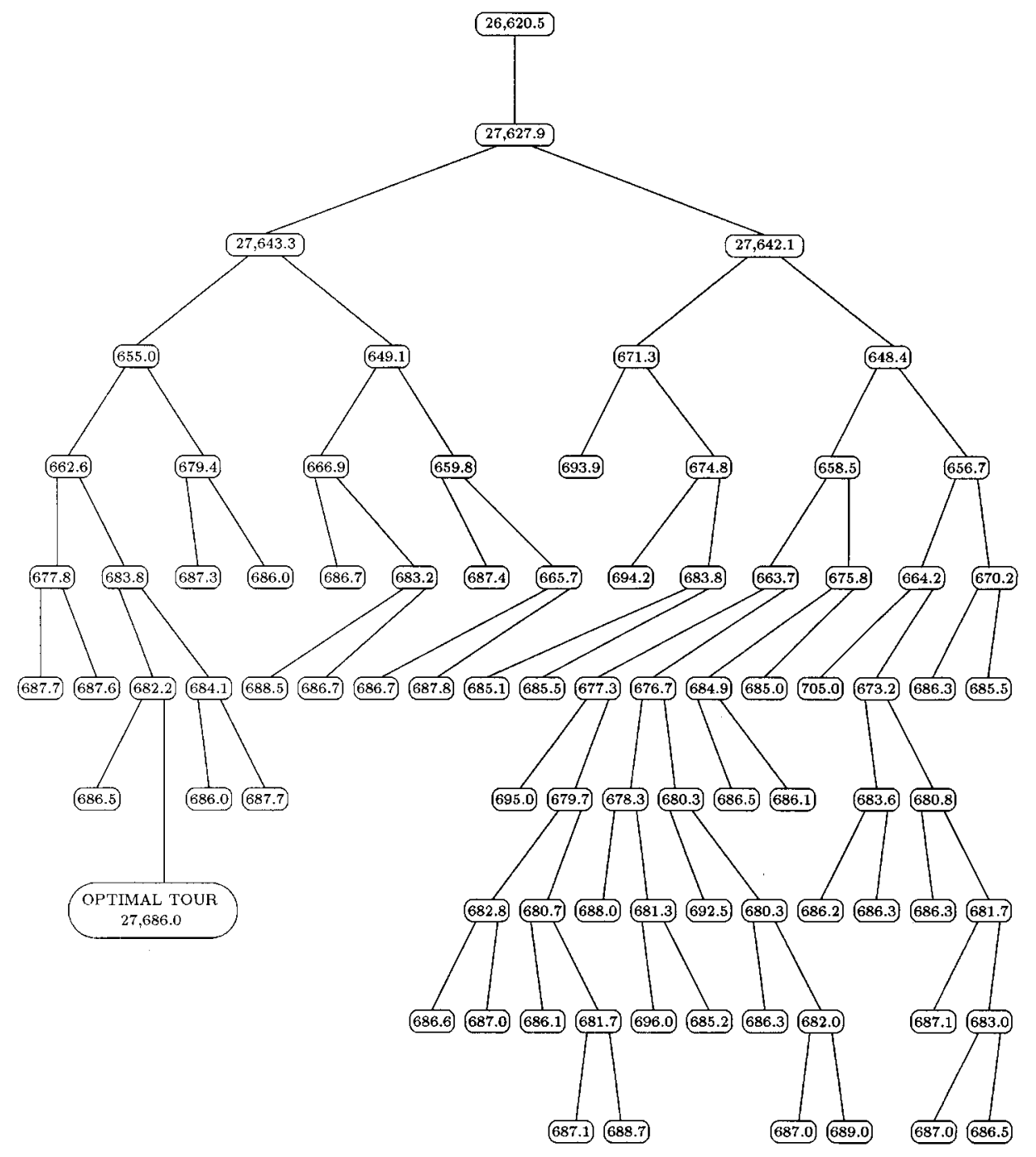

Fig. 16. Branch-and-cut tree for the 532-city TSP.

\subsection{The vertex packing problem}

NemHAUSER and SigiSMONDi (1992) report on solving randomly generated instances of the maximum cardinality vertex packing problem. The sizes of the instances vary between 40 and 120 vertices, and for every size they consider different densities by changing the probability that an edge is in the graph between 0.1 and 0.9 . The code used by the authors was limited in the sense that the cutting plane algorithm was run only in the root node, and that only primitive branching rules were available. In Table 4 we report the results for the 0.2 density instances. In general the clique inequalities closed most of the duality gap, but for low-density graphs lifted odd-holes were also important. The test instances with medium density graphs were the most (C) VVS, 1999 
Table 4. Results for the vertex packing problem

\begin{tabular}{|c|c|c|c|c|c|c|}
\hline Vertices & $\%$ gap & $\begin{array}{l}\text { Clique } \\
\text { ineq. }\end{array}$ & $\begin{array}{l}\text { Odd-hole } \\
\text { ineq. }\end{array}$ & $\begin{array}{l}\% \text { gap } \\
\text { closed }\end{array}$ & $\begin{array}{l}\text { B\&B } \\
\text { nodes }\end{array}$ & $\begin{array}{l}\text { LP- } \\
\text { iterations }\end{array}$ \\
\hline 0 & 1 & 41 & & & & \\
\hline 60 & 13 & 203 & 36 & 92.3 & 16 & 1439 \\
\hline 80 & 21 & 369 & 33 & 80.9 & 97 & 13,352 \\
\hline 90 & 15 & 222 & 13 & 86.7 & 58 & 3649 \\
\hline 100 & 29 & 181 & 19 & 93.1 & 108 & 6631 \\
\hline 110 & 35 & 781 & 5 & 77.1 & 394 & 84,115 \\
\hline 120 & 40 & 903 & 5 & 72.5 & 251 & 35,194 \\
\hline
\end{tabular}

difficult ones to solve. For instance, some of the medium-density 120-vertex problems were not solved within 100,000 LP iterations. It seems from this study that random vertex packing problems are difficult to solve by the polyhedral approach. If we consider structured vertex packing problems however, much larger instances can be tackled as the following two applications show.

\subsubsection{Frequency assignment}

The frequency assignment problem is the problem of assigning frequencies to transmission links such that no interference occurs and such that the number of used frequencies is minimized. The frequency assigned to a specific link has to be chosen from a set depending on the link. To avoid interference we have restrictions on every pair $(i, j)$ of links that the frequencies assigned to these links should differ by at least a certain prespecified amount. The problem is modeled as a vertex packing problem using a binary variable for each feasible combination of a link and a frequency. In Table 5 we present computational results as reported by AARDAL et al. (1995). The number of variables is approximately equal to forty times the number of links giving instances of between approximately 4000 and 18,000 variables. By making heavily use of preprocessing, the number of variables is reduced by at least fifty percent. The "lower bound by branch and bound" reported in the table is obtained by partial branching, and the time reported is the time needed to verify optimum, or, in the case of the last instance, the time needed to find the feasible solution of value 16 . The computations were carried out on a HP90000/720 workstation.

Table 5. Results for the frequency assignment problem

\begin{tabular}{llllr}
\hline Links & $\begin{array}{l}\text { Lower bound by } \\
\text { clique ineq. }\end{array}$ & $\begin{array}{l}\text { Lower bound by } \\
\text { B\&B }\end{array}$ & $\begin{array}{l}\text { Best known feasible } \\
\text { value }\end{array}$ & Time (s) \\
\hline 100 & 14 & 14 & 14 & 46 \\
200 & 14 & 14 & 14 & 1925 \\
340 & 20 & 22 & 22 & 6167 \\
458 & 14 & 14 & 16 & 400 \\
\hline
\end{tabular}

(C) VVS, 1999 
Table 6. Results for the airline crew scheduling problem

\begin{tabular}{rrrrrrrr}
\hline \multicolumn{2}{c}{ Original } & \multicolumn{2}{c}{ Preprocessed } & \multicolumn{2}{c}{ Prepr. } & \multicolumn{2}{c}{ B\&B } \\
Variables & Constr. & Variables & Constr. & \multicolumn{1}{c}{$z_{L P}$} & Ineq. & nodes & $z_{I P}$ \\
\hline 5198 & 531 & 3846 & 360 & 30,494 & 0 & 1 & 30,494 \\
7292 & 646 & 5862 & 488 & 26,977 & 74 & 1 & 27,040 \\
8308 & 801 & 6235 & 521 & 53,736 & 345 & 5 & 53,839 \\
8627 & 825 & 6694 & 537 & 49,616 & 37 & 1 & 49,649 \\
148,633 & 139 & 138,951 & 139 & $1,181,590$ & 0 & 1 & $1,181,590$ \\
288,507 & 71 & 202,603 & 71 & 132,878 & 0 & 1 & 132,878 \\
$1,053,137$ & 145 & 370,642 & 90 & 9950 & 389 & 1 & 10,022 \\
\hline
\end{tabular}

Table 7. Time needed to get within certain percentages of the optimal value

\begin{tabular}{cclcc}
\hline Variables & Constraints & Time $2 \%(\mathrm{~s})$ & Time $1 \%(\mathrm{~s})$ & Time opt $(\mathrm{s})$ \\
\hline 87,482 & 36 & 225 & 298 & 2642 \\
8904 & 823 & 375 & 375 & 14,441 \\
7195 & 426 & 868 & 7443 & 139,337 \\
\hline
\end{tabular}

\subsubsection{The set partitioning problem: airline crew scheduling}

HofFMAN and PADBERG (1993) report on solving huge set partitioning problems arising in ariline crew scheduling problems. The cutting plane phase uses preprocessing techniques, and clique and lifted odd-hole inequalities. In the branch-and-cut phase a variable branching rule is used. From the reported results we have selected the instances with the largest number of variables and constraints. These results are presented in Table 6. In Table $6 z_{L P}^{\mathrm{prepr}}$ denotes the LP-value after preprocessing. Of the total time needed to solvle the various problems, by far the longest time is spent on getting within the last percent of the optimal value. In Table 7 we show for three instances how much time it takes to get within one and two percent of the optimal value, as well as the time needed to verify optimality.

\subsection{The traveling salesman problem}

The literature on computational results for the traveling salesman problem is vast, and some of the results have already been shown in Section 3. To make the progress visual, we give in Table 8 a list of "world records" with respect to the size of the instances. It should be noted that there are still some small instances unsolved, which indicates that small does not necessarily imply easy, and that large is not synonymous with difficult. The instances we report on here are all Euclidean symmetric traveling salesman problems, and they arise from applications such as finding routes through actual cities, routing of drilling machines when manufacturing printed circuit boards, and x-ray crystallography. The instances can be found in the library TSPLIB, see Reeinelt (1991). Table 8 contains information on the number of "cities" $n$ of the instances. For all instances a complete graph is assumed which means that the number of variables is equal to $\frac{1}{2} n(n-1)$. The data is obtained from the original articles, so (C) VVS, 1999 
Table 8. Computational results for the traveling salesman problem

\begin{tabular}{rrrllll}
\hline Cities & \multicolumn{1}{l}{ Root } & \multicolumn{2}{l}{ B\&B } \\
\multicolumn{1}{l}{$\begin{array}{l}z_{I P} \\
\text { nodes }\end{array}$} & Application & Year & Reported by \\
\hline 49 & 12,345 & 12,345 & 1 & map USA & 1954 & Dantzig et al. \\
120 & 6942 & 6942 & 1 & map Germany & 1980 & Grötschel \\
318 & 38,765 & 41,345 & 35 & drilling & 1980 & Crowder \& Padberg \\
532 & 27,628 & 27,686 & 85 & map USA & 1987 & Padberg \& Rinaldi \\
666 & 294,080 & 294,358 & 21 & world map & 1991 & Grötschel \& Holland \\
1002 & 258,860 & 259,045 & 13 & drilling & 1990 & Padberg \& Rinaldi \\
2392 & 378,027 & 378,032 & 3 & drilling & 1990 & Padberg \& Rinaldi \\
3038 & 137,660 & 137,694 & 287 & drilling & 1992 & Applegate et al. \\
4461 & 182,528 & 182,566 & 2092 & map Germany & 1994 & Applegate et al. \\
7397 & $23,253,123$ & $23,260,728$ & 2247 & programmable & 1994 & Applegate et al. \\
& & & logic arrays & & \\
\hline
\end{tabular}

later techniques may perform differently. For instance, for the 532-city instance we know of three different numbers reported for the total number of branch-and-cut nodes needed. To give an idea of the solution times, the 2392-city problem was solved in approximately 6 hours on a CYBER. As can be seen from Table 8 , the lower bounds in the root node are very close to the optimal value which partly explains the success of cutting plane algorithms for the symmetric traveling salesman problem. When solving large instances a very advanced implementation is necessary, see Applegate et al. (1994).

\subsection{General zero-one linear programs}

CROWDER et al. (1983) present the first computational results for large-scale zero-one linear programs. The test problems are real life instances without any apparent structure. On a set of 10 instances they show the effects of simple preprocessing techniques, and knapsack cover and $(1, k)$-configuration inequalities generated and added in the root node of the branch-and-bound tree. In the other nodes they use only reduced-cost fixing to eliminate variables. Their computational results are shown in Table 9.

Table 9. Results for general zero-one problems

\begin{tabular}{|c|c|c|c|c|c|c|c|c|c|}
\hline \multicolumn{3}{|c|}{ Original problem } & \multicolumn{3}{|c|}{ Preprocessing } & \multicolumn{2}{|c|}{ Cutting plane } & \multicolumn{2}{|c|}{$\mathrm{B} \& \mathrm{~B}$} \\
\hline Vars. & Constr. & $z_{L P}$ & Vars. & Constr. & $z_{L P}$ & Ineq. & $z_{L P}$ & Nodes & $z_{I P}$ \\
\hline 33 & 16 & 2520.6 & 33 & 16 & 2819.4 & 36 & 3065.3 & 113 & 3089.0 \\
\hline 40 & 24 & $61,796.5$ & 40 & 24 & $61,829.1$ & 29 & $61,862.8$ & 11 & $62,027.0$ \\
\hline 201 & 134 & 6875.0 & 195 & 134 & 7125.0 & 139 & 7125.0 & 1116 & 7615.0 \\
\hline 282 & 242 & $176,867.5$ & 282 & 222 & $176,867.5$ & 462 & $255,033.1$ & 1862 & $258,411.0$ \\
\hline 291 & 253 & 1705.1 & 290 & 206 & 1749.9 & 278 & 5022.7 & 87 & 5223.8 \\
\hline 548 & 177 & 315.3 & 527 & 157 & 3125.9 & 296 & 8643.5 & 36 & 8691.0 \\
\hline 1550 & 94 & 1706.5 & 1550 & 94 & 1706.5 & 94 & 1706.5 & 10 & 1708.0 \\
\hline 1939 & 109 & 2051.1 & 1939 & 109 & 2051.1 & 110 & 2051.1 & 334 & 2066.0 \\
\hline 2655 & 147 & 6532.1 & 2655 & 147 & 6532.1 & 149 & 6535.0 & 214 & 6548.0 \\
\hline 2756 & 756 & 2688.7 & 2734 & 739 & 2701.1 & 1065 & 3115.3 & 2392 & 3124.0 \\
\hline
\end{tabular}

(C) VVS, 1999 


\section{Alternative techniques}

In the last two decades there has been a remarkable development in polyhedral techniques leading to an increase in the size of many combinatorial problems that can be solved by a factor hundred. Most of the computational successes have occurred for zero-one combinatorial problems where the polytope is defined once the dimension is given, such as the traveling salesman problem. For more complex combinatorial optimization problems, and for general integer programming problems less progress has been made. Here we shall give a brief overview of other available solution techniques for solving zero-one and general integer programming problems.

If the number of variables is large compared to the number of constraints column generation may in many cases be a good alternative. It can be viewed as a dual approach to polyhedral techniques in the sense that one aims at generating the extreme points of $\operatorname{conv}(S)$ rather than its facets. Instead of solving a separation problem to generate a violated inequality we need to solve the problem of finding a column, i.e., a feasible solution that can improve the objective function. Column generation was introduced by GILMORE and GOMORY (1961) to solve the cutting stock problem. Recent applications are presented by SAVELSBERGH (1993) and VANDERBECK and WoLSEY (1994).

In Lagrangean relaxation we relax the problem by removing a subset of the constraints, different from the nonnegativity constraints. Violation of the relaxed constraints is penalized by including these constraints, with a nonnegative multiplier, in the objective function. The multipliers are then updated iteratively so as to maximize the lower bound obtained from the relaxed problem. To update the Lagrangean multipliers subgradient optimization is often used. Lagrangean relaxation was used successfully by HELD and KARP $(1970,1971)$ to solve traveling salesman problems. For further details we refer to GEOFFrion (1974), HELD et al. (1974) and Fisher (1981).

LovÁsz and SCHRIJVER (1991) considered 0-1 integer linear programming problems and proposed a procedure of increasing — or lifting — the dimension of the problem by introducing more variables and then projecting the extended formulation back onto the original space. From the projection step strong valid inequalities are obtained for the original problem. They showed that by repeating this procedure a number of times equal to the number of variables in the original space, the convex hull of feasible solutions is obtained. At the lifting step the number of variables involved are squared and the number of constraints is increased by a factor two times the number of variables. BALAs et al. (1993) developed this technique further and proved that it is sufficient to double the number of variables and constraints at the lifting step. They also related this technique to a convexification technique introduced by BALAs (1979) and used this relation to develop a class of finitely converging cutting plane algorithms, called lift-and-project algorithms, for mixed 0-1 linear programming problems.

Cook et al. (1993) presented an implementation of the generalized basis reduction algorithm by LovÁsz and SCARF (1992) for solving general integer programming (C) VVS, 1999 
problems. Basis reduction was first introduced to integer programming by $\mathrm{H}$. W. LENSTRA, JR. (1983), who showed that the problem: "does there exist a vector $x \in \mathbb{Z}^{n}$ such that $A x \leq b$ ?" can be solved in polynomial time for fixed $n$. The proof was algorithmic. One important ingredient of this algorithm is the basis reduction algorithm by Lovász as described in the article by LeNSTRA, LENSTRA and Lovász (1982). The generalized basis reduction algorithm by Lovász and Scarf generalizes the algorithm by Lovász. Both the algorithm by H. W. Lenstra, Jr., and by Lovász and Scarf are based on the same principle. It is shown that it is possible, in polynomial time, to find either an integral vector belonging to the bounded polyhedron $P=\left\{x \in \mathbb{R}^{n}: A x \leq b\right\}, \quad$ or $\quad$ an integral direction $d \in \mathbb{Z}^{n} \backslash\{0\}$ such that $\max \{d x: x \in P\}-\min \{d x: x \in P\} \leq \gamma_{n}$ where $\gamma_{n}$ depends on the dimension of $P$ only. A direction $d$ as described above is called flat. Instead of branching on variables as in conventional branch-and-bound technqiues, the flat directions are used to branch on hyperplanes $d x=t, x \in P$, where $t$ is an integer varying between $\lceil\min \{\mathrm{dx}$ : $x \in P\}\rceil$ and $\lfloor\max \{d x: x \in P\}\rfloor$. Since the direction $d$ is flat the number of subproblems created at each level of the search tree is limited by a constant depending only on $n$. Moreover, we have no more than $n$ levels in the tree.

One of the main drawbacks of polyhedral techniques, as described in Section 2, is that the separation problem based on several facet defining inequalities is hard to solve, or sometimes even hard to formulate. BoyD (1994) developed a cutting plane algorithm for general integer programming that is based on so-called Fenchel duality. The basic idea of Boyd's method is to prove that a certain point $\bar{x}$ belongs to $\operatorname{conv}(S)$ or to find a separating hyperplane, that is as far as possible from $\bar{x}$. Such a separating hyperplane is referred to as a Fenchel cut. To find a Fenchel cut one needs to maximize a piecewise linear function on a nonlinear domain. Boyd suggests different relaxations of the nonlinear domain and reports on computational experience using these relaxations to solve the test problems of CROWDER et al. (1983).

TAYUR et al. (1995) used the theory of Gröbner bases to develop a solution method to solve a difficult scheduling problem. For a more general treatment of this technique we refer to Sturmfels and Thomas (1994), and Thomas (1995). The idea behind the approach by Tayur et al. is to walk from one integer solution to another in such a way that the objective function improves at every step. The directions used in this walk are specified by the Gröbner basis associated with the problem. A Gröbner basis can be viewed as a so-called test set of integral vectors $x^{1}, \ldots, x^{N}$, depending on the constraint matrix and the objective function only. These vectors have the property that a feasible solution $x^{*}$ is optimal if and only if $c\left(x^{*}+x^{k}\right) \geq c x^{*}$ whenever $x^{*}+x^{k}, k=1, \ldots, N$ is a feasible solution.

\section{Acknowledgements}

We would like to thank David Applegate for providing data on the traveling salesman problem reported in Section 4.2. Large parts of this article were written while the first author was visiting University of California at Berkeley. Financial support provided (C) VVS, 1999 
by the late Gene Lawler, and Umesh Vazirani through grant IRI-9120074 from NSF is greatly acknowledged.

\section{References}

AARdal, K. (1998), Capacitated facility location: separation algorithms and computational experience, Mathematical Programming 81, 149-175.

Aardal, K., A. Hippolito, C. P. M. van Hoesel and B. Jansen (1995), A branch-and-cut algorithm for the frequency allocation problem, Working Paper, Tilburg University, Tilburg.

Aardal, K. and C. P. M. van Hoesel (1996), Polyhedral techniques in combinatorial optimzation I: theory, Statistica Neerlandica 50, 3-26.

Aardal, K. and C. P. M. van Hoesel (1998), Uncapacitated facility location: lifting, separation and computations (in preparation).

Aardal, K., M. Labbé, J. Leung and M. Queyranne (1996), On the two-level uncapacitated facility location problem, ORSA Journal on Computing 8, 289-301.

Aardal, K., Y. Pochet and L. A. Wolsey (1995), Capacitated facility location: valid inequalities and facets, Mathematics of Operations Research 20, 562-582.

Aardal, K. and R. Weismantel (1997), Polyhedral combinatorics, in: M. Dell'Amico, F. Maffioli, S. Martello (eds.), Annotated bibliographies in combinatorial optimization, Wiley, Chichester, 45-63.

Applegate, D., R. E. Bixby, V. Chvátal and W. Cook (1994), Finding cuts in the TSP, Preliminary report, available through anonymous ftp from netlib.att.com in the directory netlib/att/math/applegate/TSP.

Araque, J. R. (1989), Solution of a 48-city vehicle routing problem by branch-and-cut, Working Paper, Department of Applied Mathematics and Statistics, State University of New York, Stony Brook.

Araque, J. R. (1990), Lots of combs of different sizes for vehicle routing, CORE Discussion Paper 9074, Louvain-la-Neuve.

Araque, J. R., L. A. Hall and T. L. Magnanti (1990), Capacitated trees, capacitated routing and associated polyhedra, CORE Discussion Paper 9061, Louvain-la-Neuve.

Balas, E. (1975), Facets of the knapsack polytope, Mathematical Programming 8, 146-164.

Balas, E. (1979), Disjunctive programming, Annals of Discrete Mathematics 5, 3-51.

BAlas, E., S. CERIA and G. CoRnuÉJols (1993), A lift-and-project cutting plane algorithm for mixed 0-1 programs, Mathematical Programming 58, 295-324.

Balas, E., S. Ceria, G. CornuéJols and Natraj (1996a), Gomory cuts revisited, Operations Research Letters 19, 1-9.

Balas, E., S. Ceria, G. Cornuésols and G. Pataki (1996b), Polyhedral methods for the maximum clique problem in: Combinatorial Optimization. DIMACS Series in Discrete Mathematics and Theoretical Computer Science, vol 26 AMS, 1-28.

BALAs, E. and M. FischetTi (1992), The fixed-outdegree 1-arborescence polytope, Mathematics of Operations Research 17, 1001-1018.

Balas, E., M. Fischetti and W. R. Pulleyblank (1995), The precedence-constrained asymmetric traveling salesman polytope, Mathematical Programming 68, 241-265.

BALAs, E. and A. C. Ho (1980), Set covering algorithms using cutting planes, heuristics and subgradient optimization, Mathematical Programming Study 12, 37-60.

BALAS, E. and S. M. NG (1989a), On the set covering polytope: I. All the facets with coefficients in $\{0,1,2\}$, Mathematical Programming 43, 57-69.

Balas, E. and S. M. NG (1989b), On the set covering polytope: II. Lifting the facets with coefficients in $\{0,1,2\}$, Mathematical Programming 45, 1-20.

Balas, E. and M. W. Padberg (1972), On the set covering problem, Operations Research 20, $1152-1161$.

Balas, E. and W. R. Pulleyblank (1983), The perfectly matchable subgraph polytope of a bipartite graph, Networks 13, 486-516.

(c) VVS, 1999 
Balas, E. and P. Toth (1985), Branch and bound methods in: E. Lawler, J. K. Lenstra, A. H. G. Rinnooy Kan and D. B. Shmoys (eds.), Traveling salesman problem: A guided tour of combinatorial optimization, Wiley and Sons.

Balas, E. and E. Zemel (1977), Critical cutsets and of graphs and canonical facets of setpacking polytopes, Mathematics of Operations Research 2, 15-19.

BALAS, E. and E. Zemel (1978), Facets of the knapsack polytope from minimal covers, SIAM Journal on Applied Mathematics 34, 119-148.

Barahona, F., M. Grötschel and A. R. Mahjoub (1985), Facets of the bipartite subgraph polytope, Mathematics of Operations Research 10, 340-358.

Barahona, F., M. Grötschel, M. JÜnger and G. Reinelt (1988), An application of combinatorial optimization to statistical physics and circuits layout design, Operations Research 36, 493-513.

Barahona, F. and A. R. Mahjoub (1986), On the cut polytope, Mathematical Programming 36, 157-173.

Barahona, F. and A. R. Mahjoub (1989), Facets of the balanced (acyclic) induced subgraph polytope, Mathematical Programming 45, 21-33.

Barahona, F. and A. R. Mahjoub (1992), On 2-connected subgraph polytopes in: E. Balas, G. Cornúzols and R. Kannan (eds.), Proceedings of the second conference on integer programming and combinatorial optimization.

BÁrÁNY, I., T. J. VAN Roy and L. A. Wolsey (1984), Uncapacitated lot-sizing: the convex hull of solutions, Mathematical Programming Study 22, 32-43.

BAuER, P. (1997), The circuit polytope: Facets, Mathematics of Operations Research 22, $110-145$.

Bienstock, D., S. Chopra, O. GüNLÜK, and C.-Y. Tsai (1998), Minimum capacity installation for multicommodity network flows, Mathematical Programming 81, 177-199.

BIENSTOCK, D. and O. GüNLÜK (1996), Capacitated network design - polyhedral structure and computation, INFORMS Journal on Computing 8, 243-259.

Boyd, E. A. (1994), Fenchel cutting planes for integer programs, Operations Research 42, 53-64.

Brunetta, L., M. Conforti and G. Rinaldi (1997), A branch-and-cut algorithm for the equicut problem, Mathematical Programming 78, 243-263.

CAprara, A. and M. Fischetti (1996), \{0, 1\}-Chvátal-Gomory cuts, Mathematical Programming 74, 221-235.

Caprara, A. and M. Fischetti (1997), Branch-and-Cut algorithms in: M. Dell'Amico, F. Maffioli, S. Martello (eds.), Annotated bibliographies in combinatorial optimization, Wiley, Chichester, pp. 45-63.

CARR, R. (1997), Separating clique trees and bipartition inequalities having a fixed number of handles and teeth in polynomial time, Mathematics of Operations Research 22, 257-265.

Ceria, S., C. Cordier, H. Marchand and L. A. Wolsey (1998), Cutting planes for integer programs with general integer variables, Mathematical Programming 81, 201-214.

Cheng, E. and W. H. Cunningham (1997), Wheel inequalities for stable set polytopes, Mathematical Programming 77, 389-421.

Cho, D. C., E. L. Johnson, M. W. PAdberg and M. R. RAo (1983a), On the uncapacitated plant location problem. I: valid inequalities and facets, Mathematics of Operations Research $\mathbf{8}$, $579-589$.

Cho, D. C., M. W. PAdberg and M. R. Rao (1983b), On the uncapacitated plant location problem. II: facets and lifting theorems, Mathematics of Operations Research 8, 590-612.

CHOPRA, S. (1989a), On tenary problems, Mathematical Programming 45, 35-47.

Chopra, S. (1989b), On the spanning tree polyhedron, Operations Research Letters 8, 25-29.

CHOPRA, S. (1992), The k-edge connected spanning subgraph polytope in: E. Balas, G. ConnuÉsols and R. KANNAN (eds.), Proceedings of the second conference on integer programming and combinatorial optimization.

Chopra, S., E. R. Gorres and M. R. Rao (1992), Solving the Steiner tree problem on a graph using branch and cut, ORSA Journal on Computing 3, 149-156.

(c) VVS, 1999 
Chopra, S. and M. R. Rao (1993), The partition problem, Mathematical Programming 59, $87-115$.

ChOPRA, S. and M. R. RAo (1994a), The Steiner tree problem I: formulations, compositions and extensions of facets, Mathematical Programming 64, 209-229.

Chopra, S. and M. R. Rao (1994b), The Steiner tree problem II: properties and classes of facets, Mathematical Programming 64, 231-246.

Chvátal, V. (1975), On certain polytopes associated with graphs, Journal of Combinatorial Theory B 18, 138-154.

Clochard, J. M. and D. NADDEF (1993), Using path inequalities in a branch and cut code for the symmetric traveling salesman problem in: G. RinAldi and L. A. Wolsey (eds.), Proceedings of the third conference on integer programming and combinatorial optimization.

Conforti, M., M. R. Rao and A. SAssano (1990/91a), The equipartition polytope I, Mathematical Programming 49, 49-70.

Conforti, M., M. R. Rao and A. Sassano (1990/91b), The equipartition polytope II, Mathematical Programming 49, 71-90.

Constantino, M. (1998), Lower bounds in lot-sizing models: A polyhedral study, Mathematics of Operations Research 23, 101-118.

Cook, W., T. Rutherford, H. E. Scarf and D. Shallcross (1993), An implementation of the generalized basis reduction algorithm for integer programming, ORSA Journal on Computing 5, 206-212.

CornúzJols, G., M. L. Fisher and G. L. Nemhauser, (1977), On the uncapacitated location problem, Annals of Discrete Mathematics 1, 163-177.

CornuÉsols, G. and F. HARChe (1993), Polyhedral study of the capacitated vehicle routing problem, Mathematical Programming 60, 21-52.

CornuÉsols, G. and W. R. PulleyblanK (1982), The traveling salesman polytope and $\{0,2\}$ matchings, Annals of Discrete Mathematics 16, 25-55.

CornuÉJols, G. and A. SAssano (1989), On the 0,1 facets of the set covering polytope, Mathematical Programming 43, 45-56.

CornuÉsols, G. and J.-M. Thizy (1982), Some facets of the simple plant location polytope, Mathematical Programming 23 50-74.

Crowder, H. P., E. L. Johnson and M. W. PAdberg (1983), Solving large-scale zero-one linear programming problems, Operations Research 31, 803-934.

Crowder, H. and M. W. PAdBerg (1980), Solving large-scale symmetric traveling salesman problems to optimality, Management Science 26, 459-509.

Dantzig, G. B., D. R. Fulkerson and S. M. Johnson (1954), Solution of a large-scale traveling-salesman problem, Operations Research 2, 393-410.

Dantzig, G. B., D. R. Fulkerson and S. M. Johnson (1959), On a linear-programming, combinatorial approach to the traveling-salesman problem, Operations Research 7, 58-66.

De Sousa, C. C. and M. Laurent (1995), Some new classes of facets for the equicut problem, Discrete Applied Mathematics 62, 167-191.

Deza, M., M. Grötschel and M. Laurent (1992), Clique-web facets for multicut polytopes, Mathematics of Operations Research 17, 981-1000.

Deza, M. and M. Laurent (1992a), Facets for the cut cone I, Mathematical Programming 56, $121-160$.

Deza, M. and M. Laurent (1992b), Facets for the cut cone II: clique-web inequalities, Mathematical Programming 56, 161-188.

DieTrich, B. L. and L. F. Escudero (1990), Coefficient reduction for knapsack like constraints in 0-1 programs, Operations Research Letters 2, 9-14.

EDmonds, J. (1965), Maximum matching and a polyhedron with 0,1-vertices, Journal of Research of the National Bureau of Standards (B) 69, 67-72.

Edmonds, J. (1970), Submodular functions, matroids and certain polyhedra, in: R. GuY et al. (eds.), Combinatorial structures and their applications, Gordon and Breach, 69-87.

Ferreira, C. E., A.Martin, C. C. De Souza, R. Weismantel and L. A. Wolsey (1996), The node capacitated graph partitioning problem: A computational study, Mathematical Programming 74, 247-267.

(C) VVS, 1999 
Ferreira, C. E., A.Martin, C. C. De Souza, R. Weismantel and L. A. Wolsey (1998), The node capacitated graph partitioning problem: A computational study, Mathematical Programming 81, 229-256.

FischeTti, M. (1991a), Facets of the asymmetric traveling salesman polytope, Mathematics of Operations Research 16, 42-56.

FischetTi, M. (1991b), Facets of two Steiner arborescence polyhedra, Mathematical Programming 51, 401-419.

FischetTI, M. (1992), Three facet-lifting theorems for the asymmetric traveling salesman polytope, in: E. Balas, G. CoRnuésols and R. Kannan (eds.), Proceedings of the second conference on integer programming and combinatorial optimization.

FisheR, M. L. (1981), The Lagrangean relaxation method for solving integer programming problems, Management Science 27, 1-18.

FleisCher, L. and É. TARDOS (1996), Separating maximally violated comb inequalities in planar graphs, in: W. H. Cunningham, S. T. McCormick and M. QueYranne (eds.), Proceedings of the Fifth Conference on Integer Programming and Combinatorial Optimization, Lecture Notes in Computer Science 1084, 475-489.

Gamble, A. B. and W. R. Pulleyblank (1989), Forest covers and a polyhedral intersection theorem, Mathematical Programming 45, 49-58.

Geoffrion, A. M. (1974), Lagrangean relaxation for integer programming, Mathematical Programming Study 2, 82-114.

Gilmore, P. C. and R. E. Gomory (1961), A linear programming approach to the cutting-stock problem, Operations Research 9, 849-859.

Goemans, M. X. (1989), Valid inequalities and separation for mixed 0-1 constraints with variable upper bounds, Operations Research Letters 8, 315-322.

Goemans, M. X. (1992), Polyhedral description of trees and arborescences, in: E. Balas, G. CornuÉsols and R. Kannan (eds.), Proceedings of the second conference on integer programming and combinatorial optimization.

Goemans, M. X. (1995), Worst-case comparison of valid inequalities for the TSP, Mathematical Programming 69, 335-349.

Goemans, M. X. and L. A. Hall (1996), The strongest facets of the acyclic subgraph polytope are unknown, in: W. H. Cunningham, S. T. McCormick and M. Queyranne (eds.), Proceedings of the fifth conference on integer programming and combinatorial optimization, Lecture Notes in Computer Science 1084, 415-429.

Gomory, R. E. (1958), Outline of an algorithm for integer solutions to linear programs, Bulletin of the American Mathematical Society 64, 275-278.

Grötschel, M. (1980), On the symmetric traveling salesman problem: solution of a 120 city problem, Mathematical Programming Studies 12, 61-77.

Grötschel, M. and O. Holland (1985), Solving matching problems with linear programming, Mathematical Programming 33, 243-259.

GrötsChel, M. and O. Holland (1991), Solution of large-scale symmetric traveling salesman problems, Mathematical Programming 51, 141-202.

GrötsChel, M., M. JÜNGER and G. REINELT (1984), A cutting plane algorithm for the linear ordering problem, Operations Research 32, 1195-1220.

Grötschel, M., M. JÜNGeR and G. ReInelt (1985a), On the acyelic subgraph polytope, Mathematical Programming 33, 28-42.

Grötschel, M., M. JüNGeR and G. ReInelt (1985b), Facets of the linear ordering problem, Mathematical Programming 33, 43-60.

Grötschel, M., L. Lovísz and A. SchriJver (1988), Geometric algorithms and combinatorial optimization, Springer-Verlag, Berlin.

Grötschel, M., A. Martin and R. Weismantel (1993), Routing in grid graphs by cutting planes, in: G. RINALDI and L. A. Wolsey (eds.), Proceedings of the third conference on integer programming and combinatorial optimization.

Grötschel, M., A. Martin and R. Weismantel (1996), Packing Steiner trees: polyhedral investigations, Mathematical Programming 72, 101-123.

(c) VVS, 1999 
Grötschel, M., A. Martin and R. Weismantel (1997), The Steiner tree packing problem in VLSI design, Mathematical Programming 78, 265-281.

Grötschel, M., C. L. Monma and M. Stoer, (1992), Computational results with a cutting plane algorithm for designing communication networks with low-connectivity constraints, Operations Research 40, 309-330.

Grötschel, M., C. L. Monma and M. Stoer, (1995), Design of survivable networks, in: M. Ball, T. L. Magnanti, C. L. Monma and G. L. Nemhauser (eds.), Handbooks in Operations Research and Management Science, Vol. 7, Network Models, 617-672, NorthHolland, Amsterdam.

Grötschel, M. and M. W. PAdberg (1979), On the symmetric traveling salesman problem I: inequalities, Mathematical Programming 16, 265-280.

Grötschel, M. and W. G. Pulleyblank (1986), Clique tree inequalities and the symmetric traveling salesman problem, Mathematics of Operations Research 11, 537-569.

Grötschel, M. and Y. WAKABAYASHI (1989), A cutting plane algorithm for a clustering problem, Mathematical Programming 45, 59-96.

Grötschel, M. and Y. WAKABAYASHI (1990), Facets of the clique-partitioning polytope, Mathematical Programming 47, 367-387.

GrötsChel, M. and Z. WIN (1992), A cutting plane algorithm for the windy postman problem, Mathematical Programming 55, 339-358.

Gu, M. Z., G. L. Nemhauser and M. W. P. Savelsbergh (1995), Sequence independent lifting of cover inequalities, in: E. BALAS and J. CLAUSEN (eds.), Proceedings of the fourth conference on integer programming and combinatorial optimization, Lecture Notes in Computer Science vol 920. Springer, 452-461.

GuignARD, M. and K. SPIELberg (1981), Logical reduction methods in zero-one programming, Operations Research 29, 49-74.

HaLl, L. (1996), Experience with a cutting plane algorithm for the capacitated spanning tree problem, INFORMS Journal on Computing 8, 219-234.

Hall, L. A. and T. L. Magnanti (1992), A polyhedral intersection theorem for capacitated spanning trees, Mathematics of Operations Research 17, 390-410.

Hammer, P. L., E. L. Johnson and U. N. Peled (1975), Facets of regular 0-1 polytopes, Mathematical Programming 8, 179-206.

Hane, C. A., C. Barnhart, E. L.Johnson, R. E.Marsten, G. L. Nemhauser and G. Sigismondi (1995), The fleet assignment problem: solving a large-scale integer program, Mathematical Programming 70, 211-232.

HeLD, M. and R. M. KaRP (1970), The traveling-salesman problem and minimum spanning trees, Operations Research 18, 1138-1162.

Held, M. and R. M. KaRP (1971), The traveling-salesman problem and minimum spanning trees: part II, Mathematical Programming 1, 6-25.

Held, M., P. Wolfe and H. P. Crowder (1974), Validation of subgradient optimization, Mathematical Programming 6, 62-88.

van Hoesel, C. P. M., A. P. M. Wagelmans and L. A. Wolsey (1994), Polyhedral characterization of the economic lot-sizing problem with start-up costs, SIAM Journal on Discrete Mathematics 7, 141-151.

Hoffman, K. L. and M. W. PADberg (1991), Improving LP-representation of zero-one linear programs for branch-and-cut, ORSA Journal on Computing 3, 121-134.

Hoffman, K. L. and M. W. PAdberg (1993), Solving airline crew scheduling problems by branch and cut, Management Science 39, 657-682.

JÜNGER, M. (1985), Polyhedral combinatorics and the acyclic subgraph problem, in: K. Hoffman and R. Wille (eds.), Research and Exposition in Mathematics 7, Helderman Verlag, Berlin.

JÜNGER, M. and P. MutZel (1993), Solving the maximum weight planar subgraph, in: G. RinAldi and L. A. Wolsey (eds.), Proceedings of the third conference on integer programming and combinatorial optimization.

(C) VVS, 1999 
Jünger, M., G. Reinelt and S. Thienel (1992), Provably good solutions for the traveling salesman problem, Report No. 92.114, Angewandte Mathematik und Informatik, Universität zu Köln.

Jünger, M., G. ReInelt and S. Thienel (1995), Practical problem solving with cutting plane algorithms in combinatorial optimization, in: W. Cook, L. Lovász and P. SEYmour (eds.), Combinatorial optimization. DIMACS series in discrete mathematics and theoretical computer science, vol 20, AMS, 111-152.

LAurent, M. (1989), A generalization of antiwebs to independence systems and their canonical facets, Mathematical Programming 45, 97-108.

LeE, J. and J. Leung, (1993a), On the Boolean quadratic forest polytope, Working Paper Department of Management Information Systems, University of Arizona, Tucson.

LeE, J. and J. LeUNG, (1993b), A comparison between two edge-coloring formulations, Operations Research Letters 13, 215-223.

Lenstra, A. K., H. W. Lenstra, JR. and L. Lovśz (1982), Factoring polynomials with rational coefficients, Matematische Annalen 261, 515-534.

LeNSTRA, JR. H. W. (1983), Integer programming with a fixed number of variables, Mathematics of Operations Research 8, 538-548.

LeUng, J. (1994), Polyhedral structure and properties of a model for layout design, European Journal on Operational Research 77, 195-207.

Leung, J. and J. LeE (1994), More facets from fences for linear ordering and acyclic subgraph polytopes, Discrete Applied Mathematics 50, 185-200.

Leung, J. M. Y. and T. L. Magnanti (1989), Valid inequalities and facets of the capacitated plant location problem, Mathematical Programming 44, 271-291.

Lovász, L. (1979), Graph theory and integer programming, Annals of Discrete Mathematics 4 , $141-158$.

Lovász, L. (1983), Submodular functions and convexity, in: A. BACHEM, M. Grötschel and B. Korte (eds.), Mathematic programming: the state of the art, Springer.

Lovász, L. and H. E. SCARF (1992), The generalized basis reduction algorithm, Mathematics of Operations Research 17, 751-764.

Lovász, L. and A. SchriJver (1991), Cones of matrices and set-functions and 0-1 optimization, SIAM Journal on Optimization 1, 166-190.

Mitchell, J. E. and B. BorChers (1992), A primal-dual interior point cutting plane method for the linear ordering problem, Report No. 204, Rensselaer Polytechnic Institute.

Mitchell, J. E. and B. BorChers (1993), Solving real-world linear ordering problems using a primal-dual interior point cutting plane method, Report No. 207, Rensselaer Polytechnic Institute.

Müller, R. and A. S. Schulz (1996), Transitive packing, in: W. H. Cunningham, S. T. McCormick and M. QueYranne (eds.), Proceedings of the fifth conference on integer programming and combinatorial optimzation, Lecture Notes in Computer Science 1084, $430-444$.

NADDEF, D. (1992), The binested inequalities for the symmetric traveling salesman polytope, Mathematics of Operations Research 17, 882-900.

NADDEF, D. and G. Rinaldi (1991), The symmetric traveling salesman polytope and its graphical relaxation: composition of valid inequalities, Mathematical Programming 51, $359-400$.

NAdDEF, D. and G. Rinaldi (1992), The crown inequalities for the symmetric traveling salesman polytope, Mathematics of Operations Research 17, 308-326.

Nemhauser, G. L. and S. PARK (1991), A polyhedral approach to edge coloring, Operations Research Letters 10, 315-322.

Nemhauser, G. L. and G. Sigismondi (1992), A strong cutting plane/branch-and-bound algorithm for node packing, Journal of the Operational Research Society 43, 443-457.

Nemhauser, G. L. and L. E. Trotter (1974), Properties of vertex packings and independence system polyhedra, Mathematical Programming 6, 48-61.

Nemhauser, G. L. and P. H. VANCE (1994), Lifted cover facets of the 0-1 knapsack polytope with GUB constraints, Operations Research Letters 16, 255-263.

(C) VVS, 1999 
Nemhauser, G. L. and L. A. Wolsey (1988), Integer and combinatorial optimization, Wiley, New York.

Nemhauser, G. L. and L. A. Wolsey (1990), A recursive procedure to general all cuts for 0-1 mixed integer programs, Mathematical Programming 46, 379-390.

NobiLI, P. and A. SASSANo (1989), Facets and lifting procedures for the set covering polytope, Mathematical Programming 45, 111-147.

PADBERG, M. W. (1973), On the facial structure of set packing polyhedra, Mathematical Programming 5, 199-215.

Padberg, M. W. (1977), On the complexity of set packing polyhedra, in: P. L. Hammer, E. L. Johnson, B. H. Korte and G. L. Nemhauser (eds.), Studies in integer programming. Annals of Discrete Mathematics 1, 421-434.

PAdBerg, M. W. (1980), $(1, k)$-configurations and facets for packing problems, Mathematical Programming 18, 94-99.

PADBERG, M. W. (1989), The Boolean quadratic polytope: some characteristics, facets and relatives, Mathematical Programming 45, 139-172.

PAdBerg, M. W. and S. Hong (1980), On the symmetric traveling salesman problem, Mathematical Programming Studies 12, 78-107.

Padberg, M. W. and G. Rinaldi (1987), Optimization of a 532-city symmetric traveling salesman polytope by branch and cut, Operations Research Letters 6, 1-7. [Addendum 9 (1990), 353.]

Padberg, M. W. and G. Rinaldi (1990), Facet identification for the symmetric traveling salesman problem, Mathematical Programming 47, 219-257.

PADBERG, M. W. and G. RinAldi (1991), A branch-and-cut algorithm for the resolution of large-scale symmetric traveling salesman problems, SIAM Review 33, 60-100.

Padberg, M. W., T. J. Van Roy and L. A. Wolsey (1985), Valid inequalities for fixed charge problems, Operations Research 33, 842-861.

Peled, U. N. (1977), Properties of facets of binary polytopes, Annals of Discrete Mathematics 1, 435-455.

Pochet, Y. and R. Weismantel (1998), The sequential knapsack polytope, SIAM Journal on Optimization 8, 248-264.

Pochet, Y. and L. A. Wolsey (1992), Network design with divisible capacities: aggregated flow and knapsack subproblems, in: E. BALAs, G. CoRnuÉJols and R. KANNAN (eds.), Proceedings of the second conference on integer programming and combinatorial optimization.

Pochet, Y. and L. A. Wolsey (1995), Algorithms and reformulations for lot sizing problems in: W. Cook, L. Lovísz and P. Seymour (eds.), Combinatorial optimization, DIMACS series in discrete mathematics and theoretical computer science, vol 20, 245-293.

Pulleyblank, W. R. and F. B. Shepherd (1993), Formulations for the stable set polytope of a claw-free graph, in: G. Rinald and L. A. Wolsey (eds.), Proceedings of the third conference on integer programming and combinatorial optimization.

Queyranne, M. and A. S. Schulz (1994), Polyhedral approaches to machine scheduling, Working Paper No. 408/1994, Fachbereich 3 Mathematik, Technische Universität Berlin, Berlin.

ReINELT, G. (1985), The linear ordering problem: algorithms and applications, Helderman, Berlin.

ReINELt, G. (1991), TSPLIB - A traveling salesman library, ORSA Journal on Computing 3, 376-384.

VAN Roy, T. J. and L. A. Wolsey (1985), Valid inequalities and separation for uncapacitated fixed charge networks, Operations Research Letters 4, 105-112.

VAN Roy, T. J. and L. A. Wolsey (1986), Valid inequalities for mixed 0-1 programs, Discrete Applied Mathematics 14, 199-213.

VAN Roy, T. J. and L. A. Wolsey (1987), Solving mixed integer programming problems using automatic reformulation, Operations Research 35, 45-57.

SAssano, A. (1989), On the facial structure of the set covering problem, Mathematical Programming 44, 181-202.

(C) VVS, 1999 
SAVELSBergh, M. W. P. (1993), A branch-and-price algorithm for the generalized assignment problem, Report COC-93-02, Computational Optimization Center, Georgia Institute of Technology, Atlanta.

Savelsbergh, M. W. P. (1994), Preprocessing and probing techniques for mixed integer programming, ORSA Journal on Computing 6, 445-454.

SCHRIJER, A. (1986), Theory of linear and integer programming, Wiley, Chichester.

Sturmfels, B. and R. R. Thomas (1994), Variation of cost functions in integer programming, Manuscript, Cornell University, Ithaca.

Thomas, R. R. (1995), A geometric Buchberger algorithm for integer programming, Mathematics of Operations Research 20, 864-884.

TAYur, S. R., R. R. Thomas and N. R. Natraj (1995), An algebraic geometry algorithm for scheduling in presence of setups and correlated demands, Mathematical Programming 69, 369-401.

TrotTer, L. (1975), A class of facet-producing graphs for vertex packing polytopes, Discrete Mathematics 12, 373-388.

VANDERBECK, F. and L. A. Wolsey (1994), An exact algorithm for IP column generation, CORE Discussion Paper 9419, Louvain-la-Neuve.

Weismantel, R. (1997), On the 0/1 knapsack polytope, Mathematical Programming 77, 49-68.

Wolsey, L. A. (1975), Faces for a linear inequality in 0-1 variables, Mathematical Programming 8, 165-178.

Wolsey, L. A. (1976a), Facets and strong valid inequalities for integer programs, Operations Research 24, 367-372.

WolseY, L. A. (1976b), Further facet generating procedures for vertex packing polytopes, Mathematical Programming 11, 158-163.

ZEMel, E. (1978), Lifting the facets of zero-one polytopes, Mathematical Programming 15, 268-277.

Received: December 1996. Revised: June 1998. 